\title{
The reconstruction of a solely time-dependent load in a simply supported non-homogeneous Euler-Bernoulli beam
}

\author{
Marijke Grimmonprez ${ }^{\mathrm{a}}$, Liviu Marin ${ }^{\mathrm{b}, \mathrm{c}, \mathrm{d}}$, Karel Van Bockstal ${ }^{\mathrm{a}, *}$ \\ ${ }^{a}$ Research Group NaM², Department of Mathematical Analysis, Ghent University, Krijgslaan 281, 9000 Ghent, Belgium \\ ${ }^{b}$ Department of Mathematics, Faculty of Mathematics and Computer Science, University of Bucharest, 14 Academiei, 010014 \\ Bucharest, Romania \\ ${ }^{c}$ Research Institute of the University of Bucharest (ICUB), University of Bucharest, 34-36 Bd. Kogălniceanu, 050107 Bucharest, \\ Romania \\ ${ }^{d}$ Institute of Mathematical Statistics and Applied Mathematics, Romanian Academy, 13 Calea 13 Septembrie, 050711 Bucharest,
} Romania

\begin{abstract}
In this paper, the theoretical and numerical determination of a solely time-dependent load distribution is investigated for a simply supported non-homogeneous Euler-Bernoulli beam. The missing source is recovered from an additional "local" integral measurement. The existence and uniqueness of a solution to the corresponding variational problem is proved by employing Rothe's method. This method also reveals a time-discrete numerical scheme based on the backward Euler method to approximate the solution. Corresponding error estimates are proved and assessed by two numerical experiments.
\end{abstract}

Keywords: inverse problem, fourth-order problem, hyperbolic problem, unknown source, time discretization, error estimates.

2010 MSC: 47J35, 65M12, 65M32

\section{Introduction}

This paper deals with the reconstruction of a solely time-dependent source function $h$ in a fourth-order hyperbolic problem that models the dynamic vibration of a simply supported non-homogeneous EulerBernoulli beam. More precisely, the problem under investigation reads as:

\footnotetext{
${ }^{*}$ Corresponding author

Email addresses: marijke.grimmonprez@ugent.be (Marijke Grimmonprez), marin.liviu@gmail.com, liviu.marin@fmi.unibuc.ro (Liviu Marin), karel.vanbockstal@ugent.be (Karel Van Bockstal)

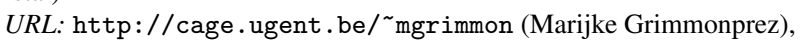
https://sites.google.com/site/marinliviu/home/(Liviu Marin), http://users. ugent.be/ kavbocks/ (Karel Van Bockstal)
} 
Determine $\{h(t), u(x, t)\}$ such that

$$
\left\{\begin{aligned}
\rho(x) \ddot{u}(x, t)+\mu(x) \dot{u}(x, t)+\left(k(x) u^{\prime \prime}(x, t)\right)^{\prime \prime}-T_{r}(x) u^{\prime \prime}(x, t) & =h(t) f(x), & & (x, t) \in(0, l) \times(0, T], \\
u(0, t)=u(l, t) & =0, & & t \in(0, T], \\
k(0) u^{\prime \prime}(0, t)=k(l) u^{\prime \prime}(l, t) & =0, & & t \in(0, T], \\
u(x, 0) & =u_{0}(x), & & x \in(0, l), \\
\dot{u}(x, 0) & =v_{0}(x), & & x \in(0, l),
\end{aligned}\right.
$$

using the integral measurement

$$
m(t)=\int_{0}^{l} \omega(x) u(x, t) \mathrm{d} x, \quad t \in[0, T],
$$

with $\omega \in \mathrm{C}_{0}^{\infty}((0, l))$. Here, $u$ is the displacement function, $u_{0}$ is the initial deflection, $v_{0}$ is the initial velocity, whilst $f(x)$ and $h(t)$ are the given (known) spatial and the unknown temporal load distributions, respectively. Further, $k=E I$, in which $E$ is the elasticity modulus and $I$ stands for the moment of inertia of the cross-section, $\rho$ is the mass density of the beam, $\mu$ is the damping coefficient per unit length and $T_{r}$ is the traction force along the beam. Note that the space and time partial derivatives of a function $z:(0, T) \times(0, l) \rightarrow \mathbb{R}$ have been denoted herein by $z^{\prime}$ and $\dot{z}$, respectively.

\subsection{Literature overview}

Important studies in the literature are devoted to source identification problems in the case of EulerBernoulli equations from either boundary or final in time observations, see e.g. [1-8]. Nicaise and Zair [1] have shown, by employing the spectral theory, that the point source $a(x)$ can be uniquely determined in the constant coefficient dynamic Euler-Bernoulli equation $\ddot{u}+u^{(i v)}=\lambda(t) a(x)$, provided that $\lambda \in C^{1}([0, T])$ is known. It should be mentioned that the missing information has been compensated by considering as an additional boundary measurement either $u^{\prime}(0, t)$ for all $t \in(0, T)$, or $u^{\prime \prime}(0, t)$ for all $t \in(0, T)$. Kawano [4] has reconsidered the aforementioned source identification problem for a more general Euler-Bernoulli equation that includes a constant damping and a constant traction force. Liu [3] has proposed the reconstruction of an unknown space and time dependent load in a constant coefficient Euler-Bernoulli beam vibration equation via an effective combination of the Lie-group adaptive method and the differential quadrature method. Hasanov [2] has investigated the recovery of the unknown source term $F(x, t)$ in a variable coefficient Euler-Bernoulli equation $\rho(x) \ddot{u}+\left(k(x) u^{\prime \prime}\right)^{\prime \prime}=F(x, t)$, by employing the least-squares method (or the quasi-solution approach), in conjunction with the adjoint problem approach, from either $u(x, T)$ or $u_{t}(x, T)$ considered as an additional measurement. The author also proved that the uniqueness of a solution can be obtained, provided that a positive condition on the solution holds [2, Lemma 7.2]. The theory developed in [2] has later been applied by Hasanov [5] to the problem of determining the unknown spatial load $f(x)$ in a cantilever beam of the form $\rho(x) \ddot{u}+\left(k(x) u^{\prime \prime}\right)^{\prime \prime}=f(x) h(t)$ from the final displacement observation. Two additional inverse source problems, namely the identification of asynchronously distributed spatial loads in the Euler-Bernoulli beam equation $\rho(x) \ddot{u}+\mu(x) \dot{u}+\left(k(x) u^{\prime \prime}\right)^{\prime \prime}-T_{r} u^{\prime \prime}=\sum_{m=1}^{M} h_{m}(t) f_{m}(x)$ with hinged-clamped 
ends, have been considered and carefully investigated by Hasanov in [6]. More specifically, $\left(f_{1}, \ldots, f_{M}\right)$ have been determined from the measured deflection in a neighborhood of a finite set of points in the first identification problem, whereas $\left(f_{1}, \ldots, f_{M}\right)$ have been determined from the measured slope in a neighborhood of the same set of points in the second identification problem. It should be mentioned that the solutions corresponding to the two aforementioned inverse source problems have been obtained using the Tikhonov regularization method, i.e. by minimizing a certain cost functional. The same method has been applied in [7] to the inverse problem of reconstructing the temporal load distribution $h(t)$ and that of recovering the spatial load distribution $f(x)$ in a vibrating beam $\rho(x) \ddot{u}+\left(k(x) u^{\prime \prime}\right)^{\prime \prime}=f(x) h(t)$ from the boundary observation $u^{\prime}(0, t)$. Recently, Van Bockstal [8] studied the determination of a spatial load distribution in a vibrating beam or plate from the final state. The author proved the uniqueness for the inverse source problem and used the Landweber method for the numerical approximation of the solution.

\subsection{New aspects}

Whilst the focus in the literature is mainly on the determination of space-dependent sources, we concentrate on this contribution to the determination of a solely time-dependent source, which is a different type of inverse problem. Usually, the unknown sources are determined by minimizing certain Tikhonov cost functionals, but we use a decoupling technique based on Rothe's method. This technique starts with a transformation of the variational formulation of the inverse problem with unknowns $u$ and $h$ into a variational problem in which the unknown $h$ is written in terms of the unknown $u$. This approach has already been successfully applied to inverse problems containing parabolic or hyperbolic second-order partial differential equations that have been studied for example in [9-14]. According to our knowledge, it is the first time that this technique is used for fourth-order operators. It is important to note that the fourth-order differential operator in (1) makes the analysis definitely more complex and challenging, even in the one-dimensional case considered herein. The numerical schemes developed in [9-14] are based on the following additional integral-type measurement

$$
\int_{\Omega} u(\boldsymbol{x}, t) \mathrm{d} \boldsymbol{x}=m(t), \quad \forall t \in[0, T] .
$$

Using the measurement above, we are not able to reconstruct the temporal load distribution in the problem under investigation because we are not able to control the fourth-order space derivative of the displacement. This is in fact the reason why the "local" measurement (2) is considered. By transfering the derivatives to the function $\omega$, this local measurement makes it possible to prove the global in time existence and uniqueness of a solution to the problem, which is a new result. The function $\omega$ then represents a smoothened characteristic function reflecting the part of the domain or the position in the domain at which the measurement is taken.

\subsection{Motivation}

In this subsection, we present a physical background of the problem under consideration. If $T_{r}$ is constant, the equation in (1) is the one-dimensional Euler-Bernoulli beam equation that models the dynamic 
vibration of a straight elastic non-homogeneous Euler-Bernoulli beam under a transverse space- and timedependent load and a horizontally applied constant traction force. The beam is oriented so that its endpoints are located at $x=0$ and $x=l$. As mentioned before, the vertical deflection of the beam at time $t$ and position $x$ is denoted by $u(x, t)$. The function $h(t)$ represents the variation in time of the vertically applied dynamic force and $f(x)$ is its variation in intensity (in force per unit length of the beam) along the beam. Alternatively, $f(x)$ is a characteristic function that reflects the position at which the transverse load is applied and $h(t)$ is its variation in intensity over the time (in force per unit length of the beam). The boundary conditions in (1) express that the beam has a pinned, roller or rocker support at both ends and that the horizontal movements are supposed to be very small such that the endpoints of the beam can be considered to be fixed. Consequently, no deflection occurs at the endpoints of the beam and the external bending moment is assumed to be zero at both ends. Such kind of beams are, for example, both end pinned beams and simply supported beams.

In the inverse problem under consideration, we look for the variation in time $h(t)$ of the applied dynamic transverse load with variation in intensity $f(x)$ and the deflection of the beam at every time $t$ and position $x$ in the beam by using a local measurement of the deflection at every time $t \in[0, T]$, given by (2]. Alternatively, we look for the variation in intensity over the time of a dynamic transverse load whose distribution is given by $f(x)$, using the same measurement.

\subsection{Goals and outline}

The first goal is to address the global (in time) solvability of the inverse source problem (12)-(2). We start with a presentation of a variational formulation of problem (1)-(2) in Section 2 . Next, in Section 3 , we discretize the problem in time by using the backward Euler's method and we prove some a priori estimates. The existence and uniqueness of a solution to the variational problem is then shown in Section 4 under appropriate conditions on the data. This method immediately reveals a constructive and convergent timediscrete numerical scheme to approximate this unique weak solution. The second goal of this contribution is to prove some error estimates for the time discretization. This error analysis is carried out in Section 5 The third goal is to compare the theoretically proven convergence rates to those obtained from some numerical experiments in Section 6. Finally, Section 7 presents the conclusions drawn from the results obtained herein.

Remark. An additional given source $F(x, t)$ can be considered in the right-hand side of the partial differential equation in (1).

\section{Variational formulation}

Taking into account the Dirichlet boundary condition, we shall work with the test space

$$
V:=\left\{\varphi \in \mathrm{H}^{2}(0, l) \mid \varphi(0)=\varphi(l)=0\right\}=\mathrm{H}^{2}(0, l) \cap \mathrm{H}_{0}^{1}(0, l) .
$$


The norm in this space is given by $\left\|\varphi^{\prime \prime}\right\|=:\|\varphi\|_{V}$ and is equivalent to the natural norm in $\mathrm{H}^{2}(0, l)$, i.e. $\|\varphi\|_{2,2}:=\sqrt{\|\varphi\|^{2}+\left\|\varphi^{\prime}\right\|^{2}+\left\|\varphi^{\prime \prime}\right\|^{2}}$, cf. [10, Theorem 1], which is in turn equivalent to $\|\varphi\|+\left\|\varphi^{\prime \prime}\right\|$, see [15, Theorem 1.8]. We shall look for a solution $\{h, u\} \in \mathrm{L}^{2}(0, T) \times \mathrm{L}^{2}((0, T), V)$. To obtain an expression for the unknown source function $h$ in terms of the unknown function $u$ and the data, we multiply the partial differential equation (PDE) in (1) by $\frac{\omega}{\rho}$ and integrate the result over $(0, l)$. We obtain

$$
(\ddot{u}(t), \omega)+\left(\frac{\mu}{\rho} \dot{u}(t), \omega\right)+\left(\left(k u^{\prime \prime}\right)^{\prime \prime}(t), \frac{\omega}{\rho}\right)-\left(T_{r} u^{\prime \prime}(t), \frac{\omega}{\rho}\right)=h(t)\left(\frac{f}{\rho}, \omega\right) .
$$

In doing this, we have to assume that

$$
\rho(x)>0, \quad \forall x \in[0, l]
$$

which is a natural assumption for the mass density. Applying the Green theorem twice to the fourth term and four times to the third term on the left-hand side (LHS) of the previous equation, we obtain

$$
(\ddot{u}(t), \omega)+\left(\frac{\mu}{\rho} \dot{u}(t), \omega\right)+\left(u(t),\left(k\left(\frac{\omega}{\rho}\right)^{\prime \prime}\right)^{\prime \prime}\right)-\left(u(t),\left(\frac{T_{r} \omega}{\rho}\right)^{\prime \prime}\right)=h(t)\left(\frac{f}{\rho}, \omega\right) .
$$

From now on, we assume that

$$
\left(\frac{f}{\rho}, \omega\right)=\int_{0}^{l} \frac{f(x) \omega(x)}{\rho(x)} \mathrm{d} x \neq 0 .
$$

If we take into account the measurement $m$, we then obtain the so-called measured problem (MP):

$$
h(t)=\frac{\ddot{m}(t)+\left(\frac{\mu}{\rho} \dot{u}(t), \omega\right)+\left(u(t),\left(k\left(\frac{\omega}{\rho}\right)^{\prime \prime}\right)^{\prime \prime}\right)-\left(u(t),\left(\frac{T_{r} \omega}{\rho}\right)^{\prime \prime}\right)}{\left(\frac{f}{\rho}, \omega\right)} .
$$

In a similar manner, we now multiply the PDE in (1) by a test function $\varphi \in V$, integrate the result over $(0, l)$ and apply Green's theorem twice to the third term on the LHS of the result. We obtain the following variational formulation:

Find $\{h, u\} \in \mathrm{L}^{2}(0, T) \times \mathrm{L}^{2}((0, T), V)$, with $\dot{u} \in \mathrm{L}^{2}\left((0, T), \mathrm{L}^{2}(0, l)\right)$ and $\ddot{u} \in \mathrm{L}^{2}\left((0, T), V^{*}\right)$, such that

$$
\begin{aligned}
& (\rho \ddot{u}(t), \varphi)+(\mu \dot{u}(t), \varphi)+\left(k u^{\prime \prime}(t), \varphi^{\prime \prime}\right)-\left(T_{r} u^{\prime \prime}(t), \varphi\right)=h(t)(f, \varphi), \\
& u(x, 0)=u_{0}(x), \quad x \in(0, l), \\
& \dot{u}(x, 0)=v_{0}(x), \quad x \in(0, l),
\end{aligned}
$$

for almost all $t \in(0, T]$ and all $\varphi \in V$, with $h(t)$ given by $(\underline{\mathrm{MP}})$.

Remark. In the following, the values $C, \varepsilon$ and $C_{\varepsilon}$ are considered to be generic and positive constants (independent of the discretization parameter), with $\varepsilon$ arbitrarily small and $C_{\varepsilon}$ arbitrarily large, i.e. $C_{\varepsilon}=$ $C\left(\frac{1}{\varepsilon}\right)$. The same notation for different constants is used, but the meaning should be clear from the context. 


\section{Time discretization and a priori estimates}

In this section, we present a time-discrete numerical scheme for problem $(\mathrm{MP})-(\mathrm{P})$. We discretize the time interval $[0, T]$ into $n \in \mathbb{N}$ equidistant subintervals $\left[t_{i-1}, t_{i}\right]$ with length $\tau=\frac{T}{n}$. The approximation of $\{h, u\}$ at time $t=t_{i}, 0 \leqslant i \leqslant n$, is denoted by $\left\{h_{i}, u_{i}\right\}$. Moreover, we approximate $\dot{u}\left(t_{i}\right), 1 \leqslant i \leqslant n$, by the backward Euler finite-difference formula $\delta u_{i}=\left(u_{i}-u_{i-1}\right) / \tau$ and $\ddot{u}\left(t_{i}\right), 1 \leqslant i \leqslant n$, by $\left(\delta u_{i}-\delta u_{i-1}\right) / \tau$. Finally, for any function $z \neq u$, we introduce the following notations

$$
z_{i}=z_{i}(x) \approx z\left(x, t_{i}\right), 0 \leqslant i \leqslant n, \quad \delta z_{i}=\frac{z_{i}-z_{i-1}}{\tau}, 1 \leqslant i \leqslant n, \quad \text { and } \quad \delta^{2} z_{i}=\frac{\delta z_{i}-\delta z_{i-1}}{\tau}, 1 \leqslant i \leqslant n .
$$

The discrete variational problem then reads as:

Find $\left\{h_{i}, u_{i}\right\} \in \mathbb{R} \times V, i=1,2, \ldots, n$, such that

$$
\begin{aligned}
& \left(\rho \delta^{2} u_{i}, \varphi\right)+\left(\mu \delta u_{i}, \varphi\right)+\left(k u_{i}^{\prime \prime}, \varphi^{\prime \prime}\right)-\left(T_{r} u_{i}^{\prime \prime}, \varphi\right)=h_{i}(f, \varphi), \\
& u_{0}(x)=u(0, x), \quad x \in(0, l), \\
& \delta u_{0}(x)=v_{0}(x), \quad x \in(0, l),
\end{aligned}
$$

for all $\varphi \in V$, with

$$
h_{i}=\frac{\ddot{m}_{i}+\left(\frac{\mu}{\rho} \delta u_{i-1}, \omega\right)+\left(u_{i-1},\left(k\left(\frac{\omega}{\rho}\right)^{\prime \prime}\right)^{\prime \prime}\right)-\left(u_{i-1},\left(T_{r} \frac{\omega}{\rho}\right)^{\prime \prime}\right)}{\left(\frac{f}{\rho}, \omega\right)} .
$$

At $t=0$, we set

$$
h_{0}:=h(0)=\frac{\ddot{m}_{0}+\left(\frac{\mu}{\rho} v_{0}, \omega\right)+\left(u_{0},\left(k\left(\frac{\omega}{\rho}\right)^{\prime \prime}\right)^{\prime \prime}\right)-\left(u_{0},\left(T_{r} \frac{\omega}{\rho}\right)^{\prime \prime}\right)}{\left(\frac{f}{\rho}, \omega\right)} .
$$

The elliptic equation (DPi) can also be written as

$$
a\left(u_{i}, \varphi\right)=\left\langle F_{i}, \varphi\right\rangle, \quad \forall \varphi \in V
$$

with

$$
a\left(u_{i}, \varphi\right):=\left(\rho \frac{u_{i}}{\tau^{2}}, \varphi\right)+\left(\mu \frac{u_{i}}{\tau}, \varphi\right)+\left(k u_{i}^{\prime \prime}, \varphi^{\prime \prime}\right)-\left(T_{r} u_{i}^{\prime \prime}, \varphi\right)
$$

and

$$
\left\langle F_{i}, \varphi\right\rangle:=h_{i}(f, \varphi)+\left(\rho \frac{u_{i-1}}{\tau^{2}}, \varphi\right)+\left(\rho \frac{\delta u_{i-1}}{\tau}, \varphi\right)+\left(\mu \frac{u_{i-1}}{\tau}, \varphi\right)
$$

The resulting numerical algorithm in pseudo-code is given below. 
Input: $T>0, n \in \mathbb{N}$, and the functions $f, \rho, \mu, k, T_{r}, m, \ddot{m}, u_{0}$ and $v_{0}$

Output: the source function $h$ and the solution $u$ at discrete time steps

Step 1. $\tau \leftarrow T / n$;

Step 2. $\theta \leftarrow[0: \tau: T]$;

Step 3. $h \leftarrow \operatorname{zeros}(n+1)$;

Step 4. $u[0] \leftarrow u_{0}$;

Step 5. $h[0] \leftarrow \frac{\ddot{m}_{0}+\left(\frac{\mu}{\rho} v_{0}, \omega\right)+\left(u_{0},\left(k\left(\frac{\omega}{\rho}\right)^{\prime \prime}\right)^{\prime \prime}\right)-\left(u_{0},\left(T_{r} \frac{\omega}{\rho}\right)^{\prime \prime}\right)}{\left(\frac{f}{\rho}, \omega\right)}$;

Step 6. For $i=1$ to $n$ do

$$
\begin{aligned}
& h[i] \leftarrow \frac{\ddot{m}_{i}+\left(\frac{\mu}{\rho} \delta u_{i-1}, \omega\right)+\left(u_{i-1},\left(k\left(\frac{\omega}{\rho}\right)^{\prime \prime}\right)^{\prime \prime}\right)-\left(u_{i-1},\left(T_{r} \frac{\omega}{\rho}\right)^{\prime \prime}\right)}{\left(\frac{f}{\rho}, \omega\right)} ; \\
& u[i] \leftarrow \operatorname{solveEP}\left(a\left(u_{i}, \varphi\right)=\left\langle F_{i}, \varphi\right\rangle\right) .
\end{aligned}
$$

The well-posedness of problem (DPi)-(DMPi) under appropriate assumptions on the data is stated in the following lemma.

Lemma 1. Suppose that $\rho(x) \geqslant \rho_{0}>0, k(x) \geqslant k_{0}>0, \mu(x) \geqslant 0$ for all $x \in[0, l]$ and $\left(\frac{f}{\rho}, \omega\right) \neq 0$. Moreover, assume that $0<T<+\infty, u_{0} \in \mathrm{L}^{2}(0, l), v_{0} \in \mathrm{L}^{2}(0, l), f \in \mathrm{L}^{2}(0, l), m \in \mathrm{C}^{2}([0, l]), \rho \in \mathrm{C}^{4}([0, l])$, $\mu \in \mathrm{C}([0, l]), k \in \mathrm{C}^{2}([0, l])$ and $T_{r} \in \mathrm{C}^{2}([0, l])$. Then positive constants $C$ and $\tau_{0}$ exist such that for any $\tau<\tau_{0}$ and $i=1,2, \ldots, n$, a unique couple $\left\{h_{i}, u_{i}\right\} \in \mathbb{R} \times V$ exists, satisfying (DPi) and (DMPi).

Proof. First, it is easy to see that the LHS of (3) defines a continuous coercive bilinear form on $V$. The bilinearity immediately follows from the bilinearity of the inner product in $\mathrm{L}^{2}(0, l)$. Moreover, the continuity is clear from

$$
|a(u, v)| \leqslant \frac{1}{\tau^{2}}\|\rho u\|\|v\|+\frac{1}{\tau}\|\mu u\|\|v\|+\left\|k u^{\prime \prime}\right\|\left\|v^{\prime \prime}\right\|+\left\|T_{r} u^{\prime \prime}\right\|\|v\| \leqslant C(\tau)\|u\|_{V}\|v\|_{V}
$$

Furthermore, it holds that

$$
\begin{aligned}
a(u, u) & =\frac{1}{\tau^{2}}(\rho u, u)+\frac{1}{\tau}(\mu u, u)+\left(k u^{\prime \prime}, u^{\prime \prime}\right)-\left(T_{r} u^{\prime \prime}, u\right) \geqslant \frac{\rho_{0}}{\tau^{2}}\|u\|^{2}+k_{0}\left\|u^{\prime \prime}\right\|^{2}-\varepsilon\left\|u^{\prime \prime}\right\|^{2}-C_{\varepsilon}\|u\|^{2} \\
& \geqslant\left(\frac{\rho_{0}}{\tau^{2}}-C_{\varepsilon}\right)\|u\|^{2}+\left(k_{0}-\varepsilon\right)\left\|u^{\prime \prime}\right\|^{2} .
\end{aligned}
$$

If we now fix $\varepsilon<k_{0}$, we obtain for every $\tau<\sqrt{\frac{\rho_{0}}{C_{\varepsilon}}}=: \tau_{0}$ that

$$
a(u, u) \geqslant C(\tau)\|u\|_{V}^{2},
$$

from which the coercivity of $a(\cdot, \cdot)$ follows. Secondly, it can be easily derived for the right-hand side (RHS) of (3) that

$$
\left|\left\langle F_{i}, \varphi\right\rangle\right| \leqslant C(\tau)\left(\left|h_{i}\right|+\left\|u_{i-1}\right\|+\left\|\delta u_{i-1}\right\|\right)\|\varphi\|_{V} .
$$


Moreover, $F_{i}$ is a linear functional on $V$, which follows from the linearity of the inner product in $\mathrm{L}^{2}(0, l)$. Now, using the Cauchy inequality and the assumptions on the data, we derive from (DMPi], for $i=1$, that

$$
\left|h_{1}\right| \leqslant C\left(\left|\ddot{m}_{1}\right|+\left\|\frac{\mu}{\rho} v_{0}\right\|\|\omega\|+\left\|u_{0}\right\|\left\|\left(k\left(\frac{\omega}{\rho}\right)^{\prime \prime}\right)^{\prime \prime}\right\|+\left\|u_{0}\right\|\left\|\left(T_{r} \frac{\omega}{\rho}\right)^{\prime \prime}\right\|\right) \leqslant C .
$$

We then obtain from (4) that $\left|\left\langle F_{1}, \varphi\right\rangle\right| \leqslant C(\tau)\|\varphi\|_{V}$ and so $F_{1}$ is a bounded linear functional on $V$. The existence of $u_{1} \in V$ solving (3) for $i=1$ is then guaranteed by the Lax-Milgram lemma. Next, we apply the following recursion for $i=2,3, \ldots, n$ :

- Step 1: Let $u_{i-2}$ and $u_{i-1} \in \mathrm{L}^{2}(0, l)$ be given. Then, analogous to the technique employed for obtaining the upper bound for $h_{1}$, DMPi) implies that $h_{i} \in \mathbb{R}$.

- Step 2: From (4) we obtain

$$
\left|\left\langle F_{i}, \varphi\right\rangle\right| \leqslant C(\tau)\left(1+\left\|u_{i-1}\right\|+\left\|\delta u_{i-1}\right\|\right)\|\varphi\|_{V} \leqslant C(i, \tau)\|\varphi\|_{V} .
$$

Thus the Lax-Milgram lemma implies the existence of $u_{i} \in V$ solving (3) for $i=1, \ldots, n$.

In what follows, some a priori estimates are proved. These are required to ensure the existence of a solution to problem $(\mathrm{MP})-(\mathrm{P})$, prove the convergence of the approximations towards that solution and to derive error estimates.

Lemma 2. Let the assumptions of Lemma 1 be fulfilled. Moreover, assume that $u_{0} \in V$. Then positive constants $\tau$ and $C$ exist such that for any $\tau<\tau_{0}$ and for every $j=1,2, \ldots, n$, the following relations hold:

(i) $\left\|\delta u_{j}\right\|^{2}+\left\|u_{j}^{\prime \prime}\right\|^{2}+\sum_{i=1}^{j}\left\|\delta u_{i}-\delta u_{i-1}\right\|^{2}+\sum_{i=1}^{j}\left\|u_{i}^{\prime \prime}-u_{i-1}^{\prime \prime}\right\|^{2} \leqslant C+C \sum_{i=1}^{j}\left|h_{i}\right|^{2} \tau$,

(ii) $\left\|u_{j}\right\|^{2} \leqslant C+C \sum_{i=1}^{j}\left|h_{i}\right|^{2} \tau$,

(iii) $\max _{1 \leqslant j \leqslant n}\left|h_{j}\right|^{2} \leqslant C$.

Proof. (i) We set $\varphi=\delta u_{i} \tau$ in (DPi) and sum it up for $i=1, \ldots, j$, keeping $1 \leqslant j \leqslant n$. We obtain

$$
\sum_{i=1}^{j}\left(\rho \delta^{2} u_{i}, \delta u_{i}\right) \tau+\sum_{i=1}^{j}\left(\mu \delta u_{i}, \delta u_{i}\right) \tau+\sum_{i=1}^{j}\left(k u_{i}^{\prime \prime}, \delta u_{i}^{\prime \prime}\right) \tau-\sum_{i=1}^{j}\left(T_{r} u_{i}^{\prime \prime}, \delta u_{i}\right) \tau=\sum_{i=1}^{j} h_{i}\left(f, \delta u_{i}\right) \tau .
$$

To the first and third term on the LHS of [5] we apply Abel's lemma [16], which states that

$$
2 \sum_{i=1}^{j} a_{i}\left(a_{i}-a_{i-1}\right)=a_{j}^{2}-a_{0}^{2}+\sum_{i=1}^{j}\left(a_{i}-a_{i-1}\right)^{2}, \quad \forall a_{i} \in \mathbb{R} .
$$

Hence, we obtain

$$
\sum_{i=1}^{j}\left(\rho \delta^{2} u_{i}, \delta u_{i}\right) \tau=\frac{1}{2}\left(\left\|\sqrt{\rho} \delta u_{j}\right\|^{2}-\left\|\sqrt{\rho} v_{0}\right\|^{2}+\sum_{i=1}^{j}\left\|\sqrt{\rho} \delta u_{i}-\sqrt{\rho} \delta u_{i-1}\right\|^{2}\right)
$$


and

$$
\sum_{i=1}^{j}\left(k u_{i}^{\prime \prime}, \delta u_{i}^{\prime \prime}\right) \tau=\frac{1}{2}\left(\left\|\sqrt{k} u_{j}^{\prime \prime}\right\|^{2}-\left\|\sqrt{k} u_{0}^{\prime \prime}\right\|^{2}+\sum_{i=1}^{j}\left\|\sqrt{k} u_{i}^{\prime \prime}-\sqrt{k} u_{i-1}^{\prime \prime}\right\|^{2}\right),
$$

respectively. Moreover, for the second term on the LHS of (5), we derive that

$$
\sum_{i=1}^{j}\left(\mu \delta u_{i}, \delta u_{i}\right) \tau=\sum_{i=1}^{j}\left\|\sqrt{\mu} \delta u_{i}\right\|^{2} \tau \geqslant 0 .
$$

Next, a combination of the Young inequality, the boundedness of $T_{r}$ and the regularity of $f$ yields

$$
\left|\sum_{i=1}^{j}\left(T_{r} u_{i}^{\prime \prime}, \delta u_{i}\right) \tau\right| \leqslant C \sum_{i=1}^{j}\left\|u_{i}^{\prime \prime}\right\|^{2} \tau+C \sum_{i=1}^{j}\left\|\delta u_{i}\right\|^{2} \tau
$$

and

$$
\left|\sum_{i=1}^{j} h_{i}\left(f, \delta u_{i}\right) \tau\right| \leqslant C \sum_{i=1}^{j}\left|h_{i}\right|^{2} \tau+C \sum_{i=1}^{j}\left\|\delta u_{i}\right\|^{2} \tau .
$$

Putting everything together and taking into account the assumptions on the data, we arrive at

$$
\begin{aligned}
(1-C \tau)\left(\left\|\delta u_{j}\right\|^{2}+\left\|u_{j}^{\prime \prime}\right\|^{2}\right)+\sum_{i=1}^{j}\left\|\delta u_{i}-\delta u_{i-1}\right\|^{2} & +\sum_{i=1}^{j}\left\|u_{i}^{\prime \prime}-u_{i-1}^{\prime \prime}\right\|^{2} \\
& \leqslant C\left(1+\sum_{i=1}^{j}\left|h_{i}\right|^{2} \tau+\sum_{i=1}^{j-1}\left\|u_{i}^{\prime \prime}\right\|^{2} \tau+\sum_{i=1}^{j-1}\left\|\delta u_{i}\right\|^{2} \tau\right) .
\end{aligned}
$$

If we now take $\tau$ strictly smaller than $\tau_{0}=\frac{1}{C+1}>0$ (i.e. $1-C \tau>1-C \tau_{0}$ ), we obtain that

$$
\begin{aligned}
& \left\|\delta u_{j}\right\|^{2}+\left\|u_{j}^{\prime \prime}\right\|^{2}+\sum_{i=1}^{j}\left\|\delta u_{i}-\delta u_{i-1}\right\|^{2}+\sum_{i=1}^{j}\left\|u_{i}^{\prime \prime}-u_{i-1}^{\prime \prime}\right\|^{2} \\
& \leqslant \frac{C}{\min \left\{1,1-C \tau_{0}\right\}}\left[1+\sum_{i=1}^{j}\left|h_{i}\right|^{2} \tau+\sum_{i=1}^{j-1}\left(\left\|u_{i}^{\prime \prime}\right\|^{2}+\left\|\delta u_{i}\right\|^{2}+\sum_{k=1}^{i}\left\|\delta u_{k}-\delta u_{k-1}\right\|^{2}+\sum_{k=1}^{i}\left\|u_{k}^{\prime \prime}-u_{k-1}^{\prime \prime}\right\|^{2}\right) \tau\right]
\end{aligned}
$$

Finally, an application of the discrete Grönwall lemma leads to $\left(\tilde{C}:=\frac{C}{\min \left\{1,1-C \tau_{0}\right\}}=\frac{C}{1-C \tau_{0}}\right)$

$$
\begin{aligned}
\left\|\delta u_{j}\right\|^{2}+\left\|u_{j}^{\prime \prime}\right\|^{2}+\sum_{i=1}^{j}\left\|\delta u_{i}-\delta u_{i-1}\right\|^{2}+\sum_{i=1}^{j}\left\|u_{i}^{\prime \prime}-u_{i-1}^{\prime \prime}\right\|^{2} & \\
& \leqslant \tilde{C}\left(1+\sum_{i=1}^{j}\left|h_{i}\right|^{2} \tau\right)+\tilde{C}^{2} \exp (\tilde{C} \tau j) \sum_{i=1}^{j-1}\left(1+\sum_{k=1}^{i}\left|h_{k}\right|^{2} \tau\right) \tau,
\end{aligned}
$$

from which the desired result follows.

(ii) This is a consequence of

$$
u_{j}=u_{0}+\sum_{i=1}^{j} \delta u_{i} \tau, \quad 1 \leqslant j \leqslant n,
$$

and the result of (i). 
(iii) Using the triangle and Cauchy inequalities, the assumptions on the data and the results of (i) and (ii), we derive from (DMPi) that

$$
\left|h_{j}\right|^{2} \leqslant C\left(1+\left\|\delta u_{j-1}\right\|^{2}+\left\|u_{j-1}\right\|^{2}\right) \leqslant C\left(1+\sum_{i=1}^{j-1}\left|h_{i}\right|^{2} \tau\right) .
$$

Applying the discrete Grönwall lemma, we conclude the proof.

Corollary 1. Let the assumptions of Lemma 2 be fulfilled. Then positive constants $C$ and $\tau_{0}$ exist such that for every $\tau<\tau_{0}$, the following relations hold

(i) $\max _{1 \leqslant j \leqslant n}\left\{\left\|u_{j}\right\|^{2}+\left\|u_{j}^{\prime \prime}\right\|^{2}+\left\|\delta u_{j}\right\|^{2}\right\}+\sum_{i=1}^{n}\left\|\delta u_{i}-\delta u_{i-1}\right\|^{2}+\sum_{i=1}^{n}\left\|u_{i}^{\prime \prime}-u_{i-1}^{\prime \prime}\right\|^{2} \leqslant C$,

(ii) $\max _{1 \leqslant i \leqslant n}\left\|\delta^{2} u_{i}\right\|_{V^{*}} \leqslant C$.

Proof. (i) This follows immediately from Lemma2

(ii) We set $\varphi=\frac{\phi}{\rho}$, with $\phi \in V$, in $\overline{\text { DPi }}$. After rearranging the terms, we obtain

$$
\left(\delta^{2} u_{i}, \phi\right)=h_{i}\left(f, \frac{\phi}{\rho}\right)+\left(T_{r} u_{i}^{\prime \prime}, \frac{\phi}{\rho}\right)-\left(\mu \delta u_{i}, \frac{\phi}{\rho}\right)-\left(k u_{i}^{\prime \prime},\left(\frac{\phi}{\rho}\right)^{\prime \prime}\right), \quad \forall \phi \in V .
$$

The triangle and Cauchy inequalities, the assumptions on the data and the result of (i) then lead to

$$
\left|\left(\delta^{2} u_{i}, \phi\right)\right| \leqslant C\left(\|\phi\|+\left\|\phi^{\prime \prime}\right\|\right) \leqslant C\|\phi\|_{V} .
$$

This relation and the definition of the norm in $V^{*}$ imply that

$$
\left\|\delta^{2} u_{i}\right\|_{V *}=\sup _{\substack{\phi \in V \\\|\phi\|_{V} \leqslant 1}}\left(\delta^{2} u_{i}, \phi\right) \leqslant C,
$$

for all $i=1, \ldots, n$, and this completes the proof.

The next a priori estimates are not required to proof the existence and uniqueness of a solution to problem $(\mathrm{P})$ - $\mathrm{MP}$. However, they can be used to increase the order of convergence of the approximations towards the unique solution, see Section 5. These a priori estimates require a definition of $\delta^{2} u_{0}$ and a variational formulation at $t=0$. Therefore, we assume that the PDE in (1) is fulfilled at $t=0$ in the space in which the RHS is defined according to the assumptions on the data, i.e.

$$
\ddot{u}(0)=\frac{1}{\rho}\left(h(0) f-\mu v(0)-\left(k u_{0}^{\prime \prime}\right)^{\prime \prime}+T_{r} u_{0}^{\prime \prime}\right) .
$$

Next, we set $\delta^{2} u_{0}:=\ddot{u}(0)$. It is easy to see that $u_{0} \in \mathrm{H}^{4}(0, l)$ and the assumptions of Lemma 2 imply that

$$
\delta^{2} u_{0} \in \mathrm{L}^{2}(0, l)
$$


Now, we multiply (7) by $\rho \varphi$, with $\varphi \in V$, and we integrate the result over $(0, l)$. After rearranging the terms, we obtain

$$
\left(\rho \delta^{2} u_{0}, \varphi\right)+\left(\mu v_{0}, \varphi\right)+\left(\left(k u_{0}^{\prime \prime}\right)^{\prime \prime}, \varphi\right)-\left(T_{r} u_{0}^{\prime \prime}, \varphi\right)=h(0)(f, \varphi), \quad \forall \varphi \in V
$$

Further, we need to assume the compatibility of the initial datum with the free bending moment boundary condition, i.e.

$$
k(0) u_{0}^{\prime \prime}(0)=k(l) u_{0}^{\prime \prime}(l)=0 .
$$

Then, applying Green's theorem twice to the third term on the LHS of 97 provides us with a variational formulation at $t=0$, i.e.

$$
\left(\rho \delta^{2} u_{0}, \varphi\right)+\left(\mu v_{0}, \varphi\right)+\left(k u_{0}^{\prime \prime}, \varphi^{\prime \prime}\right)-\left(T_{r} u_{0}^{\prime \prime}, \varphi\right)=h(0)(f, \varphi), \quad \forall \varphi \in V
$$

Note that this is exactly the same expression as $\overline{\mathrm{DPi}}$ for $i=0$. Thus $\overline{\mathrm{DPi}}$ is valid for $i=0, \ldots, n$.

Lemma 3. Let the assumptions of Lemma 2 be fulfilled. Moreover, assume that $u_{0} \in \mathrm{H}^{4}(0, l) \cap V$, $k(0) u_{0}^{\prime \prime}(0)=k(l) u_{0}^{\prime \prime}(l)=0$ and $v_{0} \in V$. Then positive constants $C$ and $\tau_{0}$ exist such that for every $\tau<\tau_{0}$ and $j \in\{1,2, \ldots, n\}$, the following relation holds

$$
\left\|\delta^{2} u_{j}\right\|^{2}+\left\|\delta u_{j}^{\prime \prime}\right\|^{2}+\sum_{i=1}^{j}\left\|\delta^{2} u_{i}-\delta^{2} u_{i-1}\right\|^{2}+\sum_{i=1}^{j}\left\|\delta u_{i}^{\prime \prime}-\delta u_{i-1}^{\prime \prime}\right\|^{2} \leqslant C+C \sum_{i=1}^{j}\left|\delta h_{i}\right|^{2} \tau
$$

Proof. First, we replace $i$ by $i-1$ in (DPi) and subtract it from (DPi). Next, we set $\varphi=\delta^{2} u_{i}$ and we sum up the result for $i=1,2, \ldots, j$, with $1 \leqslant j \leqslant n$. We obtain that

$$
\sum_{i=1}^{j}\left(\rho \delta^{3} u_{i}, \delta^{2} u_{i}\right) \tau+\sum_{i=1}^{j}\left(\mu \delta^{2} u_{i}, \delta^{2} u_{i}\right) \tau+\sum_{i=1}^{j}\left(k \delta u_{i}^{\prime \prime}, \delta^{2} u_{i}^{\prime \prime}\right) \tau-\sum_{i=1}^{j}\left(T_{r} \delta u_{i}^{\prime \prime}, \delta^{2} u_{i}\right) \tau=\sum_{i=1}^{j} \delta h_{i}\left(f, \delta^{2} u_{i}\right) \tau .
$$

If we now follow the same lines as those of the proof of Lemma 2 i) and use the regularity of the data and (8), from the previous equality we obtain

$$
\begin{aligned}
& \left\|\delta^{2} u_{j}\right\|^{2}+\left\|\delta u_{j}^{\prime \prime}\right\|^{2}+\sum_{i=1}^{j}\left\|\delta^{2} u_{i}-\delta^{2} u_{i-1}\right\|^{2}+\sum_{i=1}^{j}\left\|\delta u_{i}^{\prime \prime}-\delta u_{i-1}^{\prime \prime}\right\|^{2} \\
& \leqslant C\left(1+\left\|\delta u_{j}^{\prime \prime}\right\|^{2} \tau+\left\|\delta^{2} u_{j}\right\|^{2} \tau+\sum_{i=1}^{j-1}\left\|\delta u_{i}^{\prime \prime}\right\|^{2} \tau+\sum_{i=1}^{j-1}\left\|\delta^{2} u_{i}\right\|^{2} \tau+\sum_{i=1}^{j}\left|\delta h_{i}\right|^{2} \tau\right) .
\end{aligned}
$$

We apply the discrete Grönwall lemma to conclude the proof.

Lemma 4. Let the assumptions of Lemma 3 be fulfilled. Moreover, assume that $m \in \mathrm{C}^{3}([0, T])$. Then positive constants $C$ and $\tau_{0}$ exist such that for every $\tau<\tau_{0}$, the following inequality holds

$$
\max _{1 \leqslant j \leqslant n}\left|\delta h_{i}\right|^{2} \leqslant C
$$


Proof. First, we subtract (DMP0 from (DMPi) for $i=1$ and we divide the result by $\tau$. We obtain

$$
\delta h_{1}=\frac{\delta \ddot{m}_{1}}{\left(\frac{f}{\rho}, \omega\right)} .
$$

Using the mean value theorem (MVT) and the assumptions on the data, we immediately deduce that

$$
\left|\delta h_{1}\right| \leqslant C
$$

Next, we apply the $\delta$-operator to DMPi) for $2 \leqslant i \leqslant n$ to obtain

$$
\delta h_{i}=\frac{\delta \ddot{m}_{i}+\left(\frac{\mu}{\rho} \delta^{2} u_{i-1}, \omega\right)+\left(\delta u_{i-1},\left(k\left(\frac{\omega}{\rho}\right)^{\prime \prime}\right)^{\prime \prime}\right)-\left(\delta u_{i-1},\left(T_{r} \frac{\omega}{\rho}\right)^{\prime \prime}\right)}{\left(\frac{f}{\rho}, \omega\right)} .
$$

The triangle and Cauchy inequalities, the MVT, the assumptions on the data and the result of Corollary $11 \mathrm{i}$ ), then lead to

$$
\left|\delta h_{i}\right| \leqslant C\left(\left\|\delta^{2} u_{i-1}\right\|+\left\|\delta u_{i-1}\right\|\right) \leqslant C\left(1+\left\|\delta^{2} u_{i-1}\right\|\right)
$$

for all $i=2 \ldots, n$. From the result of Lemma 3 , it now follows that

$$
\left|\delta h_{i}\right|^{2} \leqslant C\left(1+\sum_{j=1}^{i-1}\left|\delta h_{j}\right|^{2} \tau\right)
$$

An application of the discrete Grönwall lemma then concludes the proof.

Corollary 2. Let the assumptions of Lemma 4 be fulfilled. Then positive constants $C$ and $\tau_{0}$ exist such that for every $\tau<\tau_{0}$, the following inequality holds

$$
\max _{1 \leqslant j \leqslant n}\left\{\left\|\delta^{2} u_{j}\right\|^{2}+\left\|\delta u_{j}^{\prime \prime}\right\|^{2}\right\}+\sum_{i=1}^{n}\left\|\delta^{2} u_{i}-\delta^{2} u_{i-1}\right\|^{2}+\sum_{i=1}^{n}\left\|\delta u_{i}^{\prime \prime}-\delta u_{i-1}^{\prime \prime}\right\|^{2} \leqslant C .
$$

Proof. This follows immediately from Lemmas 3 and 4

\section{Existence and uniqueness}

Now, we are ready to prove the convergence of the approximations towards a solution of $(\mathrm{MP})-(\mathrm{P})$ and to ensure the uniqueness of this solution. To do this, we first extend the approximations $h_{i}$ and $u_{i}$, $i=0, \ldots, n$, in time in a piecewise constant manner, i.e.

$$
\bar{h}_{n}:[0, T] \rightarrow \mathbb{R}, \quad t \longmapsto \begin{cases}h_{0} & t=0 \\ h_{i} & t \in\left(t_{i-1}, t_{i}\right], \quad 1 \leq i \leq n,\end{cases}
$$


and

$$
\bar{u}_{n}:(-\tau, T] \rightarrow \mathrm{L}^{2}(0, l), \quad t \longmapsto \begin{cases}u_{0} & t \in(-\tau, 0] \\ u_{i} & t \in\left(t_{i-1}, t_{i}\right], \quad 1 \leq i \leq n,\end{cases}
$$

respectively. Analogous to the case of $\bar{h}_{n}$, we denote by $\bar{m}_{n}$ the piecewise constant extension in time of $\ddot{m}_{i}=\ddot{m}\left(t_{i}\right), i=0, \ldots, n$.

Moreover, we introduce the following piecewise linear extensions in time of the same approximations:

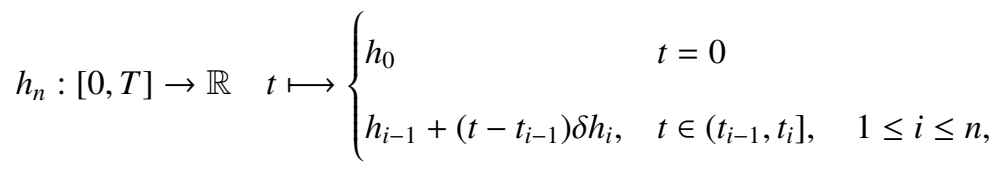

and

$$
u_{n}:(-\tau, T] \rightarrow \mathrm{L}^{2}(0, l), \quad t \longmapsto u_{i-1}+\left(t-t_{i-1}\right) \delta u_{i}, \quad t \in\left(t_{i-1}, t_{i}\right], \quad 0 \leq i \leq n,
$$

with $u_{-1}:=u_{0}+\tau \delta u_{0}$. Further, we define the step functions

$$
\bar{v}_{n}:(-\tau, T] \rightarrow \mathrm{L}^{2}(0, l), \quad t \longmapsto \begin{cases}v_{0} & t \in(-\tau, 0] \\ \delta u_{i} & t \in\left(t_{i-1}, t_{i}\right], \quad 1 \leq i \leq n\end{cases}
$$

and the piecewise linear functions in time

$$
v_{n}:(-\tau, T] \rightarrow \mathrm{L}^{2}(0, l), \quad t \longmapsto \delta u_{i-1}+\left(t-t_{i-1}\right) \delta^{2} u_{i}, \quad t \in\left(t_{i-1}, t_{i}\right], \quad 0 \leq i \leq n .
$$

Note that $\bar{v}_{n}(t)=\dot{u}_{n}(t), \forall t \in(-\tau, T]$, with $\dot{u}_{n}\left(t_{i}\right)$ defined as the left derivative of $u_{n}$ at $t_{i}$, i.e. $\dot{u}_{n}\left(t_{i}\right):=\delta u_{i}$, $i=0,1, \ldots, n$. Analogously, $\dot{v}_{n}\left(t_{i}\right):=\delta^{2} u_{i}, i=0,1, \ldots, n$. Moreover, for all $t \in(0, T]$,

$$
\bar{h}_{n}(t)=\frac{\overline{\ddot{m}}_{n}(t)+\left(\frac{\mu}{\rho} \dot{u}_{n}(t-\tau), \omega\right)+\left(\bar{u}_{n}(t-\tau),\left(k\left(\frac{\omega}{\rho}\right)^{\prime \prime}\right)^{\prime \prime}\right)-\left(\bar{u}_{n}(t-\tau),\left(\frac{T_{r} \omega}{\rho}\right)^{\prime \prime}\right)}{\left(\frac{f}{\rho}, \omega\right)} .
$$

After some simple calculations, we obtain, for any $t \in\left(t_{i-1}, t_{i}\right]$, the following relations between various Rothe functions:

$$
\begin{aligned}
& \left\|\bar{u}_{n}(t-\tau)-\bar{u}_{n}(t)\right\|+\left\|\bar{u}_{n}(t)-u_{n}(t)\right\| \leqslant\left\|u_{i}-u_{i-1}\right\|=\tau\left\|\delta u_{i}\right\|, \\
& \left\|u_{n}(t-\tau)-u_{n}(t)\right\| \leqslant \tau\left(\left\|\delta u_{i-1}\right\|+\left\|\delta u_{i}\right\|\right), \\
& \left\|\bar{u}_{n}^{\prime \prime}(t)-u_{n}^{\prime \prime}(t)\right\| \leqslant\left\|u_{i}^{\prime \prime}-u_{i-1}^{\prime \prime}\right\|=\tau\left\|\delta u_{i}^{\prime \prime}\right\|, \\
& \left\|\dot{u}_{n}(t-\tau)-\dot{u}_{n}(t)\right\|+\left\|\bar{v}_{n}(t)-v_{n}(t)\right\| \leqslant\left\|\delta u_{i}-\delta u_{i-1}\right\|=\tau\left\|\delta^{2} u_{i}\right\| .
\end{aligned}
$$

The discrete variational problem in terms of the Rothe functions then reads as:

For all $t \in(0, T]$ and $\varphi \in V$, it holds that

$$
\begin{aligned}
& \left(\rho \dot{v}_{n}(t), \varphi\right)+\left(\mu \dot{u}_{n}(t), \varphi\right)+\left(k \bar{u}_{n}^{\prime \prime}(t), \varphi^{\prime \prime}\right)-\left(T_{r} \bar{u}_{n}^{\prime \prime}(t), \varphi\right)=\bar{h}_{n}(t)(f, \varphi), \\
& u_{n}(x, 0)=u_{0}(x), \quad x \in(0, l), \\
& \dot{u}_{n}(x, 0)=v_{0}(x), \quad x \in(0, l),
\end{aligned}
$$


with $\bar{h}_{n}(t)$ given by $\overline{\mathrm{DMP}}$.

In the next theorem, we pass to the limit in $(\overline{\mathrm{DP}})-(\overline{\mathrm{DMP}})$ as $\tau \rightarrow 0$ to obtain $(\overline{\mathrm{P}})-(\overline{\mathrm{MP}})$. Note that the convergence in the proof is valid for subsequences. However, because of the uniqueness of a solution to problem $(\mathrm{P})-(\mathrm{MP})$, see Theorem 2, the convergence holds for the whole sequences of Rothe functions.

Theorem 1. Let the assumptions of Lemma 2 be fulfilled. Then there exists a couple

$$
\{h, u\} \in \mathrm{L}^{2}(0, T) \times \operatorname{Lip}\left([0, T], \mathrm{L}^{2}(0, l)\right) \cap \mathrm{L}^{\infty}((0, T), V),
$$

with $\dot{u} \in \mathrm{L}^{\infty}\left((0, T), \mathrm{L}^{2}(0, l)\right)$ and $\ddot{u} \in \mathrm{L}^{2}\left((0, T), V^{*}\right)$, such that

(i) $u_{n} \rightarrow u$ in $\mathrm{C}\left([0, T], \mathrm{L}^{2}(0, l)\right), \bar{u}_{n} \rightarrow u$ in $\mathrm{L}^{2}\left((0, T), \mathrm{L}^{2}(0, l)\right), u_{n}(t) \rightarrow u(t)$ in $V$ for all $t \in[0, T]$, $\bar{u}_{n}(t) \rightarrow u(t)$ in $V$ for all $t \in[0, T]$, and $\dot{u}_{n} \rightarrow \dot{u}$ in $\mathrm{L}^{2}\left((0, T), \mathrm{L}^{2}(0, l)\right)$;

(ii) $\bar{v}_{n} \rightarrow \dot{u}$ in $\mathrm{L}^{2}\left((0, T), \mathrm{L}^{2}(0, l)\right), v_{n} \rightarrow \dot{u}$ in $\mathrm{L}^{2}\left((0, T), \mathrm{L}^{2}(0, l)\right)$ and $v_{n} \rightarrow \ddot{u}$ in $\mathrm{L}^{2}\left((0, T), V^{*}\right)$;

(iii) $\bar{h}_{n} \rightarrow h$ in $\mathrm{L}^{2}((0, T))$;

(iv) $\{h, u\}$ solves problem $(\mathbb{P}$;

(v) $\{h, u\}$ solves problem $\mathrm{MP}$.

Proof. (i) From Corollary 1 (i), it follows that

$$
\max _{t \in[0, T]}\left\|\dot{u}_{n}(t)\right\|^{2}+\max _{t \in[0, T]}\left\|\bar{u}_{n}(t)\right\|_{V}^{2} \leqslant C .
$$

Due to the embedding $V \hookrightarrow \hookrightarrow \mathrm{L}^{2}(\Omega)$, it follows that all assumptions of [17, Lemma 1.3.13] are satisfied. Therefore, there exist a function $u \in \operatorname{Lip}\left([0, T], \mathrm{L}^{2}(0, l)\right) \cap \mathrm{L}^{\infty}((0, T), V)$ with $\dot{u} \in \mathrm{L}^{\infty}\left((0, T), \mathrm{L}^{2}(0, l)\right)$, and a subsequence of $\left\{u_{n}\right\}_{n \in \mathbb{N}}$ (denoted by the same symbol again) such that

$$
\left\{\begin{array}{lll}
u_{n} \rightarrow u & \text { in } \quad \mathrm{C}\left([0, T], \mathrm{L}^{2}(0, l)\right), \\
u_{n}(t)-u(t) & \text { in } \quad V, \quad \forall t \in[0, T], \\
\bar{u}_{n}(t)-u(t) & \text { in } \quad V, \quad \forall t \in[0, T], \\
\dot{u}_{n} \rightarrow \dot{u} & \text { in } \quad \mathrm{L}^{2}\left((0, T), \mathrm{L}^{2}(0, l)\right) .
\end{array}\right.
$$

Now, (12) and Corollary 1 i) lead to

$$
\left\|\bar{u}_{n}(t)-u_{n}(t)\right\| \leqslant C \tau .
$$

Consequently, $\left\{u_{n}\right\}_{n \in \mathbb{N}}$ and $\left\{\bar{u}_{n}\right\}_{n \in \mathbb{N}}$ have the same limit in $\mathrm{L}^{2}\left((0, T), \mathrm{L}^{2}(0, l)\right)$ and hence

$$
\bar{u}_{n} \rightarrow u \quad \text { in } \quad \mathrm{L}^{2}\left((0, T), \mathrm{L}^{2}(0, l)\right) .
$$

(ii) Since $\bar{v}_{n}=\dot{u}_{n}$, we immediately get from (i) that

$$
\bar{v}_{n} \rightarrow \dot{u} \quad \text { in } \quad \mathrm{L}^{2}\left((0, T), \mathrm{L}^{2}(0, l)\right) .
$$


Moreover, Corollary[1ii) yields

$$
\int_{0}^{T}\left\|v_{n}(t)-\bar{v}_{n}(t)\right\|^{2} \leqslant \sum_{i=1}^{n}\left\|\delta u_{i}-\delta u_{i-1}\right\|^{2} \tau \leqslant C \tau .
$$

This actually means that $\left\{v_{n}\right\}_{n \in \mathbb{N}}$ and $\left\{\bar{v}_{n}\right\}_{n \in \mathbb{N}}$ have the same limit in $\mathrm{L}^{2}\left((0, T), \mathrm{L}^{2}(0, l)\right)$ as $\tau \rightarrow 0$. Hence, it also holds true that

$$
v_{n} \rightarrow \dot{u} \quad \text { in } \quad \mathrm{L}^{2}\left((0, T), \mathrm{L}^{2}(0, l)\right)
$$

Further, Corollary 1 (ii) and the reflexivity of the space $\mathrm{L}^{2}\left((0, T), V^{*}\right)$ guarantee the existence of a function $w \in \mathrm{L}^{2}\left((0, T), V^{*}\right)$ and a subsequence of $\left\{\dot{v}_{n}\right\}_{n \in \mathbb{N}}$ (denoted by the same symbol again) such that

$$
\dot{v}_{n} \rightarrow w \quad \text { in } \quad \mathrm{L}^{2}\left((0, T), V^{*}\right) .
$$

From [18, Proposition 23.9] it follows that $w=\ddot{u}$ in $\mathrm{L}^{2}\left((0, T), V^{*}\right)$ and therefore

$$
\dot{v}_{n} \rightarrow \ddot{u} \quad \text { in } \quad \mathrm{L}^{2}\left((0, T), V^{*}\right) .
$$

(iii) Lemma2 2 iii) ensures the boundedness of $\bar{h}_{n}$ in the reflexive space $\mathrm{L}^{2}(0, T)$. Consequently, there exist a function $h \in \mathrm{L}^{2}(0, T)$ and a subsequence of $\left\{\bar{h}_{n}\right\}_{n \in \mathbb{N}}$ (denoted by the same symbol again) such that

$$
\bar{h}_{n} \rightarrow h \quad \text { in } \quad \mathrm{L}^{2}(0, T) \text {. }
$$

(iv) We integrate (DP) over $(0, \eta), \eta \in(0, T]$, and obtain, for all $\varphi \in V$

$$
\int_{0}^{\eta}\left(\rho \dot{v}_{n}(t), \varphi\right)+\int_{0}^{\eta}\left(\mu \dot{u}_{n}(t), \varphi\right)+\int_{0}^{\eta}\left(k \bar{u}_{n}^{\prime \prime}(t), \varphi^{\prime \prime}\right)-\int_{0}^{\eta}\left(T_{r} \bar{u}_{n}^{\prime \prime}(t), \varphi\right)=\int_{0}^{\eta} \bar{h}_{n}(t)(f, \varphi) .
$$

Next, we pass to the limit in (16) as $n \rightarrow \infty$. From (i)-(iii), it follows that

$$
\int_{0}^{\eta}(\rho \dot{v}(t), \varphi)+\int_{0}^{\eta}(\mu \dot{u}(t), \varphi)+\int_{0}^{\eta}\left(k \bar{u}^{\prime \prime}(t), \varphi^{\prime \prime}\right)-\int_{0}^{\eta}\left(T_{r} \bar{u}^{\prime \prime}(t), \varphi\right)=\int_{0}^{\eta} h(t)(f, \varphi),
$$

If we differentiate this result with respect to $\eta$, we arrive at $\mathbb{P}$ for almost all $\eta \in(0, T]$. This actually means that $\{h, u\}$ solves problem $(\mathrm{P})$.

(v) We integrate (DMP) over $(0, \eta), \eta \in(0, T]$, and obtain, for all $\varphi \in V$

$$
\int_{0}^{\eta} \bar{h}_{n}(t) \mathrm{d} t=\frac{\int_{0}^{\eta}\left(\overline{\ddot{m}}_{n}(t)+\left(\frac{\mu}{\rho} \dot{u}_{n}(t-\tau), \omega\right)+\left(\bar{u}_{n}(t-\tau),\left(k\left(\frac{\omega}{\rho}\right)^{\prime \prime}\right)^{\prime \prime}\right)-\left(\bar{u}_{n}(t-\tau),\left(\frac{T_{r} \omega}{\rho}\right)^{\prime \prime}\right)\right) \mathrm{d} t}{\left(\frac{f}{\rho}, \omega\right)} .
$$

Next, we pass to the limit in (17) as $n \rightarrow \infty$. For the LHS of relation (17), from (iii) we immediately obtain

$$
\int_{0}^{\eta} \bar{h}_{n}(t) \mathrm{d} t \rightarrow \int_{0}^{\eta} h(t) \mathrm{d} t
$$

Moreover, for the first term on the RHS of relation (17) it holds that

$$
\int_{0}^{\eta} \ddot{m}_{n}(t) \mathrm{d} t \rightarrow \int_{0}^{\eta} \ddot{m}(t) \mathrm{d} t .
$$


This follows from the Lebesgue dominated theorem, the regularity of $\ddot{m}$ and the definition of $\bar{m}$ as a step function. Next, the triangle inequality leads to

$$
\begin{aligned}
\left|\int_{0}^{\eta}\left(\dot{u}_{n}(t-\tau)-\dot{u}(t), \frac{\mu}{\rho} \omega\right) \mathrm{d} t\right| & \leqslant\left|\int_{0}^{\eta}\left(\dot{u}_{n}(t-\tau)-\dot{u}_{n}(t), \frac{\mu}{\rho} \omega\right) \mathrm{d} t\right|+\left|\int_{0}^{\eta}\left(\dot{u}_{n}(t)-\dot{u}(t), \frac{\mu}{\rho} \omega\right) \mathrm{d} t\right| \\
& =: R_{1}+R_{2}
\end{aligned}
$$

and

$$
\begin{aligned}
& \left|\int_{0}^{\eta}\left(\bar{u}_{n}(t-\tau)-u(t),\left(k\left(\frac{\omega}{\rho}\right)^{\prime \prime}\right)^{\prime \prime}\right) \mathrm{d} t\right| \\
& \quad \leqslant\left|\int_{0}^{\eta}\left(\bar{u}_{n}(t-\tau)-\bar{u}_{n}(t),\left(k\left(\frac{\omega}{\rho}\right)^{\prime \prime}\right)^{\prime \prime}\right) \mathrm{d} t\right|+\left|\int_{0}^{\eta}\left(\bar{u}_{n}(t)-u(t),\left(k\left(\frac{\omega}{\rho}\right)^{\prime \prime}\right)^{\prime \prime}\right) \mathrm{d} t\right| \\
& \quad=: P_{1}+P_{2} .
\end{aligned}
$$

For $R_{1}$ and $P_{1}$ we apply the Cauchy inequality, the regularity of the data, (12), 15) and Corollary 1 (i). Consequently, we obtain

$$
R_{1} \leqslant \int_{0}^{\eta}\left(\left\|\dot{u}_{n}(t-\tau)-\dot{u}_{n}(t)\right\|\left\|\frac{\mu}{\rho} \omega\right\|\right) \mathrm{d} t \leqslant C \sum_{i=1}^{n}\left\|\delta u_{i}-\delta u_{i-1}\right\| \tau \leqslant C \sqrt{\tau}
$$

and

$$
P_{1} \leqslant \int_{0}^{\eta}\left(\left\|\bar{u}_{n}(t-\tau)-\bar{u}_{n}(t)\right\|\left\|\left(k\left(\frac{\omega}{\rho}\right)^{\prime \prime}\right)^{\prime \prime}\right\|\right) \mathrm{d} t \leqslant C \tau \sum_{i=1}^{n}\left\|\delta u_{i}\right\| \tau \leqslant C \tau .
$$

This implies that $R_{1} \rightarrow 0$ and $P_{1} \rightarrow 0$ as $n \rightarrow \infty$. Moreover, it immediately follows from (i) that $R_{2} \rightarrow 0$ and $P_{2} \rightarrow 0$ as $n \rightarrow \infty$. Collecting all limits, we obtain

$$
\int_{0}^{\eta}\left(\frac{\mu}{\rho} \dot{u}_{n}(t-\tau), \omega\right) \mathrm{d} t \rightarrow \int_{0}^{\eta}\left(\frac{\mu}{\rho} \dot{u}(t), \omega\right) \mathrm{d} t
$$

and

$$
\int_{0}^{\eta}\left(\bar{u}_{n}(t-\tau),\left(k\left(\frac{\omega}{\rho}\right)^{\prime \prime}\right)^{\prime \prime}\right) \mathrm{d} t \rightarrow \int_{0}^{\eta}\left(u(t),\left(k\left(\frac{\omega}{\rho}\right)^{\prime \prime}\right)^{\prime \prime}\right) \mathrm{d} t .
$$

Finally, we analogously deduce that

$$
\int_{0}^{\eta}\left(\bar{u}_{n}(t-\tau),\left(\frac{T_{r} \omega}{\rho}\right)^{\prime \prime}\right) \mathrm{d} t \rightarrow \int_{0}^{\eta}\left(u(t),\left(\frac{T_{r} \omega}{\rho}\right)^{\prime \prime}\right) \mathrm{d} t .
$$

Collecting all limits gives us the following limit of (17) as $n \rightarrow \infty$ :

$$
\int_{0}^{\eta} h(t) \mathrm{d} t=\frac{\int_{0}^{\eta}\left(\ddot{m}(t)+\left(\frac{\mu}{\rho} \dot{u}(t), \omega\right)+\left(u(t),\left(k\left(\frac{\omega}{\rho}\right)^{\prime \prime}\right)^{\prime \prime}\right)-\left(u(t),\left(\frac{T_{r} \omega}{\rho}\right)^{\prime \prime}\right)\right) \mathrm{d} t}{\left(\frac{f}{\rho}, \omega\right)}, \quad \forall \eta \in(0, T] .
$$

Taking the derivative with respect to $\eta$ of the above equality, we then arrive at

$$
h(\eta)=\frac{\ddot{m}(\eta)+\left(\frac{\mu}{\rho} \dot{u}(\eta), \omega\right)+\left(u(\eta),\left(k\left(\frac{\omega}{\rho}\right)^{\prime \prime}\right)^{\prime \prime}\right)-\left(u(\eta),\left(\frac{T_{r} \omega}{\rho}\right)^{\prime \prime}\right)}{\left(\frac{f}{\rho}, \omega\right)}, \quad \text { for a.a. } \eta \in(0, T] .
$$

This means that $\{h, u\}$ solves problem (MP). 
Theorem 2. Let the assumptions of Lemma 2 be fulfilled. Then the couple

$$
\{h, u\} \in \mathrm{L}^{2}(0, T) \times\left[\operatorname{Lip}\left([0, T], \mathrm{L}^{2}(0, l)\right) \cap \mathrm{L}^{\infty}((0, T), V)\right],
$$

from Theorem 1 is the only solution to problem $(\mathrm{MP}-\mathrm{P})$.

Proof. Suppose that two solution $\left\{h_{1}, u_{1}\right\}$ and $\left\{h_{2}, u_{2}\right\}$ exist, solving problem (MP)-(P). Set $u:=u_{1}-u_{2}$ and $h:=h_{1}-h_{2}$. Then $u(x, 0)=0$ and $\dot{u}(x, 0)=0$ for almost all $x \in(0, l)$. Subtract the measured problem (MP) for $\left\{h_{2}, u_{2}\right\}$ from that corresponding to $\left\{h_{1}, u_{1}\right\}$ and integrate the result over $[0, \eta], \eta \in(0, T]$, to obtain

$$
\int_{0}^{\eta} h(t) \mathrm{d} t=\frac{\left(\frac{\mu}{\rho} u(\eta), \omega\right)+\left(\int_{0}^{\eta} u(t) \mathrm{d} t,\left(k\left(\frac{\omega}{\rho}\right)^{\prime \prime}\right)^{\prime \prime}\right)-\left(\int_{0}^{\eta} u(t) \mathrm{d} t,\left(\frac{T_{r} \omega}{\rho}\right)^{\prime \prime}\right)}{\left(\frac{f}{\rho}, \omega\right)} .
$$

Using the triangle and Cauchy inequalities, we obtain, for every $\eta \in[0, T]$,

$$
\left|\int_{0}^{\eta} h(t) \mathrm{d} t\right| \leqslant C\left(\|u(\eta)\|+\left\|\int_{0}^{\eta} u(t) \mathrm{d} t\right\|\right) .
$$

Moreover, subtracting the variational problem $(\mathbb{P})$ for $\left\{h_{2}, u_{2}\right\}$ from that corresponding to $\left\{h_{1}, u_{1}\right\}$ and integrating the result over $[0, \eta], \eta \in(0, T]$, yields

$$
(\rho \dot{u}(\eta), \varphi)+(\mu u(\eta), \varphi)+\left(k \int_{0}^{\eta} u^{\prime \prime}(t) \mathrm{d} t, \varphi^{\prime \prime}\right)-\left(T_{r} \int_{0}^{\eta} u^{\prime \prime}(t) \mathrm{d} t, \varphi\right)=(f, \varphi) \int_{0}^{\eta} h(t) \mathrm{d} t .
$$

Next, we set $\varphi=u(\eta)$ in $(19)$ and integrate the result over $[0, \xi], \xi \in(0, T]$, to obtain

$$
\begin{aligned}
\frac{1}{2}\|\sqrt{\rho} u(\xi)\|^{2}+\int_{0}^{\xi}\|\sqrt{\mu} u(\eta)\|^{2} \mathrm{~d} \eta & +\frac{1}{2}\left\|\sqrt{k} \int_{0}^{\xi} u^{\prime \prime}(t) \mathrm{d} t\right\|^{2} \\
& =\int_{0}^{\xi}(f, u(\eta))\left(\int_{0}^{\eta} h(t) \mathrm{d} t\right) \mathrm{d} \eta+\int_{0}^{\xi}\left(T_{r} \int_{0}^{\eta} u^{\prime \prime}(t) \mathrm{d} t, u(\eta)\right) \mathrm{d} \eta .
\end{aligned}
$$

Using the Cauchy and Young inequalities, the assumptions on the data and (18), we successively deduce that

$$
\begin{aligned}
\|u(\xi)\|^{2}+\left\|\int_{0}^{\xi} u^{\prime \prime}(t) \mathrm{d} t\right\|^{2} & \leqslant C\left(\int_{0}^{\xi}\left|\int_{0}^{\eta} h(t) \mathrm{d} t\right|^{2} \mathrm{~d} \eta+\int_{0}^{\xi}\|u(\eta)\|^{2} \mathrm{~d} \eta+\int_{0}^{\xi}\left\|\int_{0}^{\eta} u^{\prime \prime}(t) \mathrm{d} t\right\|^{2} \mathrm{~d} \eta\right) \\
& \leqslant C\left(\int_{0}^{\xi}\|u(\eta)\|^{2} \mathrm{~d} \eta+\int_{0}^{\xi}\left\|\int_{0}^{\eta} u^{\prime \prime}(t) \mathrm{d} t\right\|^{2} \mathrm{~d} \eta\right) .
\end{aligned}
$$

An application of the Grönwall lemma then yields

$$
\|u(\xi)\|^{2}+\left\|\int_{0}^{\xi} u^{\prime \prime}(t) \mathrm{d} t\right\|^{2}=0
$$

for all $\xi \in(0, T]$. Therefore, $u=0$ a.e. in $(0, l) \times(0, T]$. From this fact and [18), it follows immediately that $\int_{0}^{\eta} h(t) \mathrm{d} t=0$ for all $\eta \in(0, T]$. Taking the derivative with respect to $\eta$ implies that $h=0$ a.e. in $(0, T]$. This concludes the proof by contradiction.

Remark. Note that the convergence of $\bar{h}_{n}$ to $h$ has only been proved in a weak sense. However, under the assumption of Lemma 4 it also holds that $\bar{h}_{n} \rightarrow h$ and $h_{n} \rightarrow h$ in $\mathrm{L}^{2}(0, T)$. This follows from Lemma 2 Lemma 4 and the Arzelà-Ascoli theorem, see [19] Theorem 1.5.3]. 


\section{Error analysis}

In this section, we prove some error estimates. We introduce the following notations:

$$
e_{u}:=u_{n}-u, \quad e_{\bar{u}}:=\bar{u}_{n}-u, \quad e_{\bar{h}}:=\bar{h}_{n}-h, \quad e_{\bar{m}}:=\overline{\ddot{m}}_{n}-\ddot{m} .
$$

The main theorem in this section, namely Theorem 3 , shows that the order of convergence of the approximations towards the unique weak solutions increases if we feature higher regularity on the data.

Lemma 5. (i) Let the regularity of the data be as in Theorem 1 Moreover, assume that $m \in \mathrm{H}^{3}(0, T)$. Then positive constants $C$ and $\tau_{0}$ exist such that for any $\tau \leqslant \tau_{0}$ and all $\eta \in[0, T]$, the following relation holds

$$
\left|\int_{0}^{\eta} e_{\bar{h}}(t) d t\right| \leqslant C\left(\sqrt{\tau}+\left\|e_{u}(\eta)\right\|+\left\|\int_{0}^{\eta} e_{u}(t) d t\right\|\right) .
$$

(ii) Let the regularity of the data be as in Theorem 1. Moreover, assume that $m \in \mathrm{C}^{3}([0, T])$. Then positive constants $C$ and $\tau_{0}$ exist such that for any $\tau \leqslant \tau_{0}$ and all $\eta \in[0, T]$, the following relation holds

$$
\left|\int_{0}^{\eta} e_{\bar{h}}(t) d t\right| \leqslant C\left(\tau+\left\|e_{u}(\eta)\right\|+\left\|\int_{0}^{\eta} e_{u}(t) d t\right\|\right) .
$$

Proof. (i) First, we subtract (MP) from (DMP) and integrate the result over $(0, \eta), \eta \in(0, T]$, to obtain

$$
\begin{aligned}
& \int_{0}^{\eta} e_{\bar{h}}(t) \mathrm{d} t=\frac{1}{\left(\frac{f}{\rho}, \omega\right)} {\left[\int_{0}^{\eta} e_{\bar{m}}(t) \mathrm{d} t+\left(\frac{\mu}{\rho}\left(u_{n}(\eta-\tau)-u(\eta)\right), \omega\right)\right.} \\
&\left.+\left(\int_{0}^{\eta}\left(\bar{u}_{n}(t-\tau)-u(t)\right) \mathrm{d} t,\left(k\left(\frac{\omega}{\rho}\right)^{\prime \prime}\right)^{\prime \prime}\right)+\left(\int_{0}^{\eta}\left(\bar{u}_{n}(t-\tau)-u(t)\right) \mathrm{d} t,\left(\frac{T_{r} \omega}{\rho}\right)^{\prime \prime}\right)\right]
\end{aligned}
$$

Next, we add $\pm u_{n}(t)$ or $\pm \bar{u}_{n}(t)$ to the appropriate terms and obtain

$$
\begin{aligned}
\int_{0}^{\eta} e_{\bar{h}}(t) \mathrm{d} t & =\int_{0}^{\eta} e_{\bar{m}}(t) \mathrm{d} t+\left(\frac{\mu}{\rho}\left(u_{n}(\eta-\tau)-u_{n}(\eta)\right), \omega\right)+\left(\frac{\mu}{\rho} e_{u}(\eta), \omega\right) \\
& +\left(\int_{0}^{\eta}\left(\bar{u}_{n}(t-\tau)-\bar{u}_{n}(t)\right) \mathrm{d} t,\left(k\left(\frac{\omega}{\rho}\right)^{\prime \prime}\right)^{\prime \prime}\right)+\left(\int_{0}^{\eta} e_{\bar{u}}(t) \mathrm{d} t,\left(k\left(\frac{\omega}{\rho}\right)^{\prime \prime}\right)^{\prime \prime}\right) \\
& +\left(\int_{0}^{\eta}\left(\bar{u}_{n}(t-\tau)-\bar{u}_{n}(t)\right) \mathrm{d} t,\left(\frac{T_{r} \omega}{\rho}\right)^{\prime \prime}\right)+\left(\int_{0}^{\eta} e_{\bar{u}}(t) \mathrm{d} t,\left(\frac{T_{r} \omega}{\rho}\right)^{\prime \prime}\right)=: \sum_{i=1}^{7} S_{i} .
\end{aligned}
$$

The Cauchy inequality and the regularity of $m$ lead to

$$
\left|S_{1}\right| \leqslant \sum_{i=1}^{n} \int_{t_{i-1}}^{t_{i}} \int_{t}^{t_{i}}|\dddot{m}(t)| \mathrm{d} t \leqslant \sum_{i=1}^{n} \int_{t_{i-1}}^{t_{i}} \sqrt{\int_{t_{i-1}}^{t_{i}}|\dddot{m}|^{2}} \sqrt{\tau} \leqslant C \sqrt{\tau}
$$

Moreover, using equation (13) and Corollary 11), we obtain

$$
\left|S_{2}\right| \leqslant C \tau \text {. }
$$


Further, equation (12) and Corollary 1 i) yield

$$
\begin{aligned}
\left|S_{3}\right|+\left|S_{5}\right|+\left|S_{7}\right| & \leqslant C\left(\left\|e_{u}\right\|+\left\|\int_{0}^{\eta} e_{\bar{u}}\right\|\right) \leqslant C\left(\left\|e_{u}\right\|+\int_{0}^{\eta}\left\|\bar{u}_{n}-u_{n}\right\|+\left\|\int_{0}^{\eta} e_{u}\right\|\right) \\
& \leqslant C\left(\tau+\left\|e_{u}\right\|+\left\|\int_{0}^{\eta} e_{u}\right\|\right)
\end{aligned}
$$

and

$$
\left|S_{4}\right|+\left|S_{6}\right| \leqslant C \tau
$$

Collecting all estimates above, we conclude the proof.

(ii) The proof is similar to that of (i), the only difference being the upper bound for $S_{1}$. The higher regularity of $m$ now leads to

$$
\left|S_{1}\right| \leqslant C \tau .
$$

Now, we can prove the following error estimates.

Theorem 3. (i) Let the regularity of the data be as in Theorem 1 Moreover, assume that $m \in \mathrm{H}^{3}(0, T)$.

Then positive constants $C$ and $\tau_{0}$ exist such that for any $\tau \leqslant \tau_{0}$, the following relation holds

$$
\max _{\eta \in[0, T]}\left|\int_{0}^{\eta} e_{\bar{h}}(t) d t\right|^{2}+\max _{\eta \in[0, T]}\left\|e_{u}(\eta)\right\|^{2}+\max _{\eta \in[0, T]}\left\|\int_{0}^{\eta} e_{u}^{\prime \prime}(t) d t\right\|^{2} \leqslant C \tau .
$$

(ii) Let the regularity of the data be as in Lemma 4 Then positive constants $C$ and $\tau_{0}$ exist such that for any $\tau \leqslant \tau_{0}$, the following relation holds

$$
\max _{\eta \in[0, T]}\left|\int_{0}^{\eta} e_{\bar{h}}(t) d t\right|^{2}+\max _{\eta \in[0, T]}\left\|e_{u}(\eta)\right\|^{2}+\max _{\eta \in[0, T]}\left\|\int_{0}^{\eta} e_{u}^{\prime \prime}(t) d t\right\|^{2} \leqslant C \tau^{2} .
$$

Proof. (i) We subtract (P) from (DP) and integrate the result over $(0, \xi), \xi \in[0, T]$, to obtain for all $\varphi \in V$

$$
\left(\rho v_{n}(\xi)-\rho \dot{u}(\xi), \varphi\right)+\left(\mu e_{u}(\xi), \varphi\right)+\left(k \int_{0}^{\xi} e_{\bar{u}}^{\prime \prime}(t) \mathrm{d} t, \varphi\right)-\left(T_{r} \int_{0}^{\xi} e_{\bar{u}}^{\prime \prime}(t) \mathrm{d} t, \varphi\right)=(f, \varphi) \int_{0}^{\xi} e_{\bar{h}}(t) \mathrm{d} t .
$$

Next, we set $\varphi=e_{u}(\xi)$, add $\pm \dot{u}_{n}(\xi)$ to the first term on the LHS of (20) and $\pm u_{n}^{\prime \prime}(t)$ to the third term on the LHS of [20, and integrate the result over $(0, \eta), \eta \in[0, T]$. After some rearrangements in the terms, we obtain that

$$
\begin{aligned}
& \int_{0}^{\eta}\left(\rho \dot{e}_{u}(\xi), e_{u}(\xi)\right) \mathrm{d} \xi+\int_{0}^{\eta}\left\|\sqrt{\mu} e_{u}(\xi)\right\|^{2} \mathrm{~d} \xi+\int_{0}^{\eta}\left(k \int_{0}^{\xi} e_{u}^{\prime \prime}(t) \mathrm{d} t, e_{u}^{\prime \prime}(\xi)\right) \mathrm{d} \xi \\
& =\int_{0}^{\eta}\left(f, e_{u}(\xi)\right)\left(\int_{0}^{\xi} e_{\bar{h}}(t) \mathrm{d} t\right) \mathrm{d} \xi-\int_{0}^{\eta}\left(\rho v_{n}(\xi)-\rho \bar{v}_{n}(\xi), e_{u}(\xi)\right) \mathrm{d} \xi \\
& \quad-\int_{0}^{\eta}\left(k \int_{0}^{\xi}\left(\bar{u}_{n}^{\prime \prime}-u_{n}^{\prime \prime}\right), e_{u}^{\prime \prime}(\xi)\right) \mathrm{d} \xi+\int_{0}^{\eta}\left(T_{r} \int_{0}^{\xi} e_{\bar{u}}^{\prime \prime}, e_{u}(\xi)\right) \mathrm{d} \xi=: \sum_{i=1}^{4} Q_{i} .
\end{aligned}
$$


The LHS of (21) simplifies to

$$
\frac{1}{2}\left\|\sqrt{\rho} e_{u}(\eta)\right\|^{2}+\int_{0}^{\eta}\left\|\sqrt{\mu} e_{u}(\xi)\right\|^{2} \mathrm{~d} \xi+\frac{1}{2}\left\|\sqrt{k} \int_{0}^{\eta} e_{u}^{\prime \prime}(t) \mathrm{d} t\right\|^{2}
$$

From the Cauchy and Young inequalities, the regularity of $f$ and Lemma 5 we obtain for $Q_{1}$

$$
\left|Q_{1}\right| \leqslant C\left(\int_{0}^{\eta}\left|\int_{0}^{\xi} e_{\bar{h}}(t) \mathrm{d} t\right|^{2} \mathrm{~d} \xi+\int_{0}^{\eta}\left\|e_{u}(\xi)\right\|^{2} \mathrm{~d} \xi\right) \leqslant C\left(\tau^{2}+\int_{0}^{\eta}\left\|e_{u}\right\|^{2}\right) .
$$

Moreover, using the boundedness of $\rho,(15)$ and Corollary 1 i), we deduce that

$$
\left|Q_{2}\right| \leqslant C\left(\int_{0}^{\eta}\left\|v_{n}-\bar{v}_{n}\right\|^{2}+\int_{0}^{\eta}\left\|e_{u}\right\|^{2}\right) \leqslant C\left(\tau \sum_{i=1}^{n}\left\|\delta u_{i}-\delta u_{i-1}\right\|^{2}+\int_{0}^{\eta}\left\|e_{u}\right\|^{2}\right) \leqslant C\left(\tau+\int_{0}^{\eta}\left\|e_{u}\right\|^{2}\right) .
$$

To obtain an upper bound for $Q_{3}$, we first apply the integration by parts formula to obtain

$$
Q_{3}=\int_{0}^{\eta}\left(k\left(u_{n}^{\prime \prime}(\xi)-\bar{u}_{n}^{\prime \prime}(\xi)\right), \int_{0}^{\xi} e_{u}^{\prime \prime}(t) \mathrm{d} t\right) \mathrm{d} \xi-\left(k \int_{0}^{\eta}\left(u_{n}^{\prime \prime}(t)-\bar{u}_{n}^{\prime \prime}(t)\right) \mathrm{d} t, \int_{0}^{\eta} e_{u}^{\prime \prime}(t) \mathrm{d} t\right) .
$$

Next, the Cauchy and Young inequalities, the boundedness of $k$, (14) and Corollary 1 (i) lead to

$$
\begin{aligned}
\left|Q_{3}\right| & \leqslant C_{\varepsilon} \int_{0}^{\eta}\left\|u_{n}^{\prime \prime}-\bar{u}_{n}^{\prime \prime}\right\|^{2}+C \int_{0}^{\eta}\left\|\int_{0}^{\xi} e_{u}^{\prime \prime}\right\|^{2}+\varepsilon\left\|\int_{0}^{\eta} e_{u}^{\prime \prime}\right\|^{2} \\
& \leqslant C_{\varepsilon} \tau \sum_{i=1}^{n}\left\|u_{i}^{\prime \prime}-u_{i-1}^{\prime \prime}\right\|^{2}+C \int_{0}^{\eta}\left\|\int_{0}^{\xi} e_{u}^{\prime \prime}\right\|^{2}+\varepsilon\left\|\int_{0}^{\eta} e_{u}^{\prime \prime}\right\|^{2} \leqslant C_{\varepsilon} \tau+C \int_{0}^{\eta}\left\|\int_{0}^{\xi} e_{u}^{\prime \prime}\right\|^{2}+\varepsilon\left\|\int_{0}^{\eta} e_{u}^{\prime \prime}\right\|^{2} .
\end{aligned}
$$

Finally, $Q_{4}$ can be bounded as

$$
\begin{aligned}
\left|Q_{4}\right| & \leqslant C\left(\int_{0}^{\eta}\left\|\int_{0}^{\xi} e_{\bar{u}}^{\prime \prime}(t) \mathrm{d} t\right\|^{2} \mathrm{~d} \xi+\int_{0}^{\eta}\left\|e_{u}(\xi)\right\|^{2} \mathrm{~d} \xi\right) \\
& \leqslant C\left(\int_{0}^{\eta}\left\|\bar{u}_{n}^{\prime \prime}-u_{n}^{\prime \prime}\right\|^{2}+\int_{0}^{\eta}\left\|\int_{0}^{\xi} e_{u}^{\prime \prime}\right\|^{2}+\int_{0}^{\eta}\left\|e_{u}\right\|^{2}\right) \leqslant C\left(\tau+\int_{0}^{\eta}\left\|\int_{0}^{\xi} e_{u}^{\prime \prime}\right\|^{2}+\int_{0}^{\eta}\left\|e_{u}\right\|^{2}\right),
\end{aligned}
$$

Collecting all of the results above, we arrive at

$$
\left\|e_{u}(\eta)\right\|^{2}+\left\|\int_{0}^{\eta} e_{u}^{\prime \prime}\right\|^{2} \leqslant C_{\varepsilon}\left(\tau+\int_{0}^{\eta}\left\|e_{u}\right\|^{2}+\int_{0}^{\eta}\left\|\int_{0}^{\xi} e_{u}^{\prime \prime}\right\|^{2}\right)+\varepsilon\left\|\int_{0}^{\eta} e_{u}^{\prime \prime}\right\|^{2} .
$$

If we now fix $\varepsilon$ small enough, an application of the Grönwall lemma gives

$$
\left\|e_{u}(\eta)\right\|^{2}+\left\|\int_{0}^{\eta} e_{u}^{\prime \prime}\right\|^{2} \leqslant C \tau .
$$

This, together with Lemma 5 , concludes the proof.

(ii) The proof follows more or less the same lines as that of (i), the main difference being the use of Corollary 2 for estimating $Q_{2}, Q_{3}$ and $Q_{4}$, such that $O\left(\tau^{2}\right)$ is obtained instead of $O(\tau)$.

Corollary 3. Let the regularity of the data be as in Theorem 3 ii). Then positive constants $C$ and $\tau_{0}$ exist such that for any $\tau \leqslant \tau_{0}$ it holds that

$$
\max _{\eta \in[0, T]}\left|\int_{0}^{\eta}\left(h_{n}-h\right)(t) d t\right|^{2} \leqslant C \tau^{2} .
$$


Proof. It holds that

$$
\begin{aligned}
\left|\int_{0}^{\eta}\left(h_{n}-h\right)(t) \mathrm{d} t\right|^{2} & \leqslant C\left(\left|\int_{0}^{\eta}\left(h_{n}-\bar{h}_{n}\right)(t) \mathrm{d} t\right|^{2}+\left|\int_{0}^{\eta}\left(\bar{h}_{n}-h\right)(t) \mathrm{d} t\right|^{2}\right) \\
& \leqslant C\left(\tau^{2} \int_{0}^{\eta}\left|\partial_{t} h_{n}(t)\right|^{2} \mathrm{~d} t+\left|\int_{0}^{\eta}\left(\bar{h}_{n}-h\right)(t) \mathrm{d} t\right|^{2}\right) \\
& \leqslant C \tau^{2},
\end{aligned}
$$

for which we have used the triangle inequality, Lemma 4 and Theorem 3 (ii).

\section{Numerical results}

\subsection{Setting}

In the following, we consider a simply supported homogeneous steel beam (ASTM-A36) of length $l$, width $b$ and height $h(l \gg b, h)$ to be specified later. The units for displacement and time are meters and seconds, respectively. The governing equations in (1)-(2) can be rearranged in a more convenient form (if we consider $f h$ for the time being as $f$ )

$$
\left\{\begin{aligned}
\ddot{U}(\tilde{x}, \tilde{t})+\dot{U}(\tilde{x}, \tilde{t})+U^{(i v)}(\tilde{x}, \tilde{t})-\sqrt{\frac{\rho}{k}} \frac{T_{r}}{\mu} U^{\prime \prime}(\tilde{x}, \tilde{t}) & =F(\tilde{x}, \tilde{t}), & & (\tilde{x}, \tilde{t}) \in\left(0, \frac{l}{\ell}\right) \times\left(0, \frac{c T}{\ell}\right], \\
U(0, \tilde{t})=U\left(\frac{l}{\ell}, \tilde{t}\right) & =0, & & \tilde{t} \in\left(0, \frac{c T}{\ell}\right], \\
U^{\prime \prime}(0, \tilde{t})=U^{\prime \prime}\left(\frac{l}{\ell}, \tilde{t}\right) & =0, & & \tilde{t} \in\left(0, \frac{c T}{\ell}\right], \\
U(\tilde{x}, 0) & =U_{0}(\tilde{x}), & & \tilde{x} \in\left(0, \frac{l}{\ell}\right), \\
\dot{U}(\tilde{x}, 0) & =V_{0}(\tilde{x}), & & \tilde{x} \in\left(0, \frac{l}{\ell}\right), \\
\int_{0}^{\frac{l}{\ell}} W(\tilde{x}) U(\tilde{x}, \tilde{t}) \mathrm{d} \tilde{x} & =M(\tilde{t}) & & \tilde{t} \in\left(0, \frac{c T}{\ell}\right],
\end{aligned}\right.
$$

by using the following nondimensional variables [8]:

$$
\begin{array}{r}
\tilde{x}=\frac{1}{\ell} x, \quad \tilde{t}=\frac{c}{\ell} t, \quad U(\tilde{x}, \tilde{t})=\frac{1}{\tilde{u}} u(x, t), \quad F(\tilde{x}, \tilde{t})=\frac{1}{\tilde{f}} f(x, t), \quad W(\tilde{x})=\omega(\ell \tilde{x}), \\
M(\tilde{t})=\frac{m\left(\frac{\ell \tilde{t}}{c}\right)}{\ell \tilde{u}}, \quad U_{0}(\tilde{x})=\frac{1}{\tilde{u}} u_{0}(\ell \tilde{x}), \quad V_{0}(\tilde{x})=\frac{\ell}{c \tilde{u}} v_{0}(\ell \tilde{x}),
\end{array}
$$

where $c=\frac{k^{1 / 4} \mu^{1 / 2}}{\rho^{3 / 4}}$ (unit: $\left.\mathrm{m} / \mathrm{s}\right), \ell=\frac{\rho c}{\mu}$ (unit: $\left.\mathrm{m}\right), \tilde{u}=\ell$ and $\tilde{f}=\frac{\rho c^{2}}{\ell}$ (unit: $\mathrm{kg} / \mathrm{s}^{2}$ ). Now, we rewrite $F$ as

$$
F(\tilde{x}, \tilde{t})=\mathcal{H}(\tilde{t}) \mathcal{F}(\tilde{x})+\mathcal{G}(\tilde{x}, \tilde{t})
$$

with $\mathcal{H}(t)$ unknown. Note that the dimensionless variables are used in the following experiments, with the mention that in the sequel the tilde sign is dropped in order to simplify the notations employed.

We assume that for the two experiments considered herein the exact displacement is prescribed as

$$
U(x, t)=0.0001(1+t)^{2} x^{2}(x-4)^{4}, \quad x \in[0,4], \quad t \in[0,1]
$$

It is assumed that the width $b$ and height $h$ are both equal to $0.01 \mathrm{~m}$, so that the vibrations of the beam can be described in a one-dimensional setting. Then, the moment of inertia (unit: $\mathrm{m}^{4}$ ) of the rectangular 
solid cross section of the beam is given by $I=\frac{b h^{3}}{12}$. The elasticity modulus $E$ is $200 \times 10^{9} \mathrm{~N} / \mathrm{m}^{2}$, so that the flexural rigidity $k$ is approximately equal to $167 \mathrm{Nm}^{2}$. The other material coefficients are given by $\rho=8 \mathrm{~kg} / \mathrm{m}, T_{r}=6 \times 10^{3} \mathrm{~N}$ and $\mu=6 \times 10^{2} \mathrm{~kg} / \mathrm{s} \times \mathrm{m}$. From the nondimensionalization of this problem, it follows that this setting corresponds to a beam of length $l \approx 1 \mathrm{~m}$ and a final time $T \approx 0.0133 \mathrm{~s}$.

In the experiments considered herein, the exact sources are given by

$$
\left(\mathcal{H}_{1}(t), \mathcal{F}_{1}(x)\right)=(\exp (t), 0.1) \quad \text { and } \quad\left(\mathcal{H}_{2}(t), \mathcal{F}_{2}(x)\right)=(4-4(\sin (2 \pi t)+\cos (2 \pi t)), 0.05),
$$

where the subindex of $\mathcal{H}$ and $\mathcal{F}$ corresponds to the number of the experiment. Four different choices for the mollifier are considered

$$
\begin{aligned}
& W_{1}(x)=\exp \left(-5(x-2)^{2}\right), \quad W_{2}(x)=\exp \left(-10(x-2)^{2}\right), \\
& W_{3}(x)=\exp \left(-50(x-2)^{2}\right), \quad W_{4}(x)=\exp \left(-100(x-2)^{2}\right),
\end{aligned}
$$

They define the position of the measurement, i.e. around $x=2$. A graphical representation is given in Figure 1. A randomly generated uncorrelated noise is added to the additional measurement in order to simulate the inherent errors present in real measurements:

$$
M_{p}(t)=M(t)(1+p \mathcal{R}(t)),
$$

where $p$ represents the percentage of noise, e.g. $p=0.01$ for $1 \%$ noise, and $\mathcal{R}$ is a random number in the interval $[-1,1]$ that changes in time. Afterwards, the noisy data is regularized by using the nonlinear least-squares method to obtain a third-order polynomial approximating the noisy data. It is this polynomial that is used in the computations.

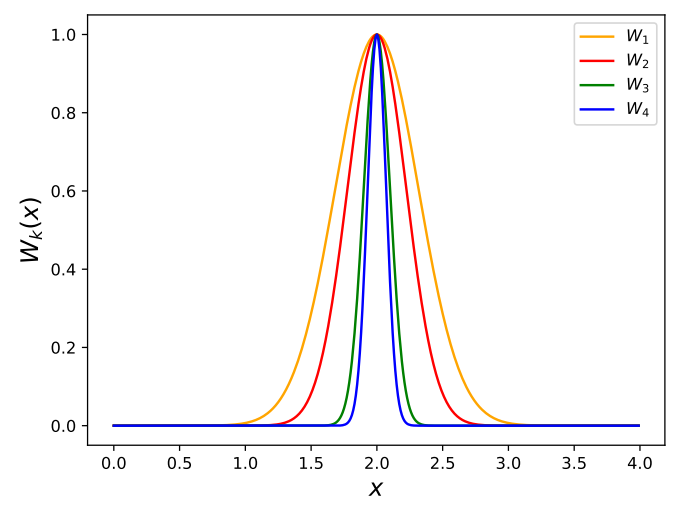

Figure 1: Various mollifiers used in the numerical experiments.

The solution to the inverse source problem is obtained by applying the algorithm proposed in Section 3 The corresponding forward problems in this algorithm are discretized in time according to the backward Euler method, with the time step to be specified later. The partial differential equation in problem 22 
is split into two second-order equations using $v=-u^{\prime \prime}$ as a second unknown. At each time step, the resulting elliptic mixed problems are solved numerically by the finite element method using the secondorder (P2-FEM) Lagrange polynomials for the space discretization, with the mention that the number of space discretization intervals is taken to be equal to 200. The finite element library DOLFIN [20, 21] from the FEniCS project [22, 23] is used to solve the forward problems.

In the following subsection, the exact value for the source is compared to its corresponding numerically retrieved value. Also, the error estimates at the final time are examined when $p=0$ with the use of the following notations

$$
E_{\mathcal{H}_{i}}(\tau)=\left|\int_{0}^{T} e_{\overline{\mathcal{H}_{i}}}(t) \mathrm{d} t\right|, i=1,2 ; \quad \text { and } \quad E_{U_{T}}(\tau)=\left\|e_{U}(T)\right\| .
$$

\subsection{Results}

The expected convergence rate of the numerical procedure is predicted in Theorem 3 ii) for a smooth measurement, i.e.

$$
E_{\mathcal{H}_{i}}(\tau), E_{U_{T}}(\tau) \sim O(\tau), i=1,2
$$

In Figures 2 23 , the errors are plotted for both experiments on a logarithmic scale, for $\tau \in\left\{2^{-j}: j=\right.$ $5, \ldots, 10\}$ and exact measurements. Then, the order of convergence corresponds to the slope of the linear regression line through the numerically obtained points. As can be seen from these figures, the numerical results confirm the theoretically obtained linear behaviour for all choices of the mollifier. We can also see that the error is smaller when the support of the mollifier is larger. A possible explanation for this is the fact that there is more information included in the case of a larger support. The CPU time (in seconds, Intel ${ }^{\circ}$ Core $^{\mathrm{TM}}$ i7-4810MQ Processor) for the different experiments is similar and can be found in Table 1 . for Experiment 1 (using the mollifier $W_{1}$ ). For the smallest time step $\tau=2^{-10}$, the results are obtained in approximately one minute.

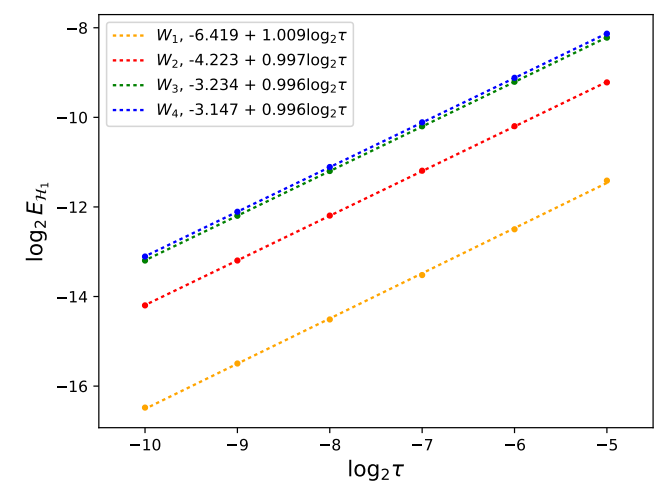

(a)

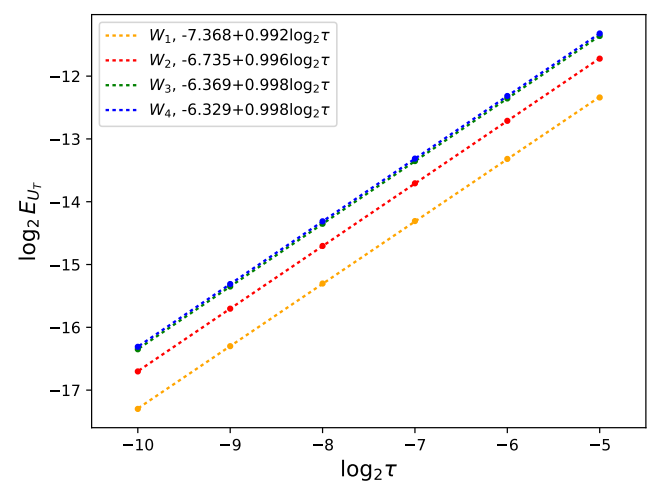

(b)

Figure 2: Convergence rate for Experiment 1 (with $p=0$ ) on a logarithmic scale: (a) $\log _{2} E_{\mathcal{H}_{1}}$, and (b) $\log _{2} E_{U_{T}}$. 
Table 1: The CPU time (in seconds) obtained for Experiment 1 (mollifier $W_{1}$ ) and various time steps.

\begin{tabular}{c|cccccc}
\hline$\tau$ & $2^{-5}$ & $2^{-6}$ & $2^{-7}$ & $2^{-8}$ & $2^{-9}$ & $2^{-10}$ \\
\hline time & 2.16 & 2.88 & 5.62 & 11.56 & 24.11 & 54.98 \\
\hline
\end{tabular}

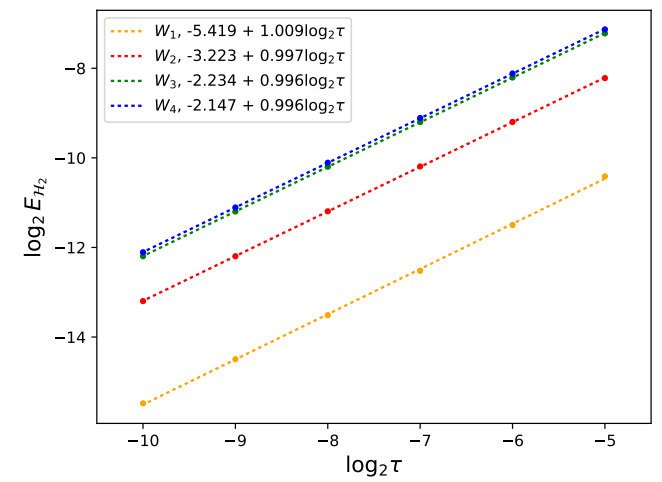

(a)

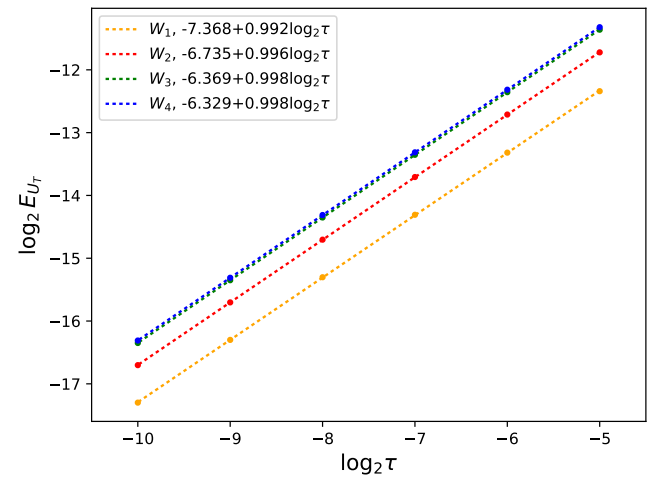

(b)

Figure 3: Convergence rate for Experiment 2 (with $p=0$ ) on a logarithmic scale: (a) $\log _{2} E_{\mathcal{H}_{2}}$, and (b) $\log _{2} E_{U_{T}}$.

In the following, for the experiments with noise added to the measurement, only the mollifiers $W_{1}$ and $W_{4}$ are considered. Moreover, the time step is fixed and equal to $\tau=2^{-8}$ in all experiments. In Figures 4 . 5. the numerical solution for Experiment 1 is compared to the exact solution $\mathcal{H}_{1}$ for various levels of noise added to the measurements, i.e. $p \in\{0.001,0.01,0.03,0.05,0.1\}$. For both mollifiers, an accurate approximation for the source $\mathcal{H}_{1}$ is obtained for a noise level less than or equal to $5 \%$. This shows the stability of the numerical procedure. Also note that the absolute error is smaller when using mollifier $W_{4}$. This is explained by the fact that in this experiment the graph of the exact measurement with the use of $W_{4}$ lies below the graph of the measurement corresponding to $W_{1}$. 


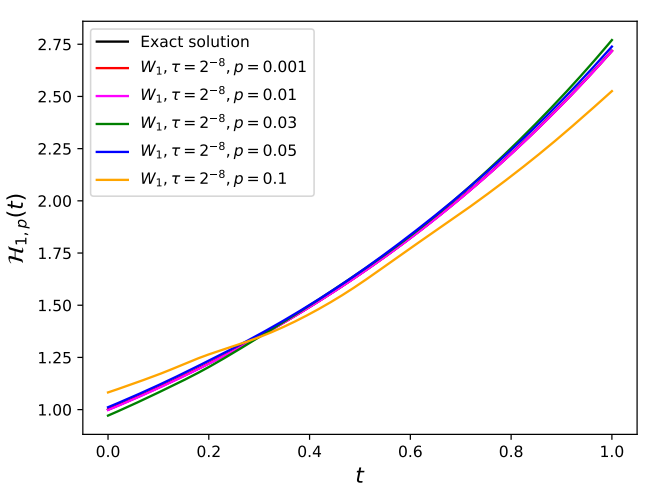

(a)

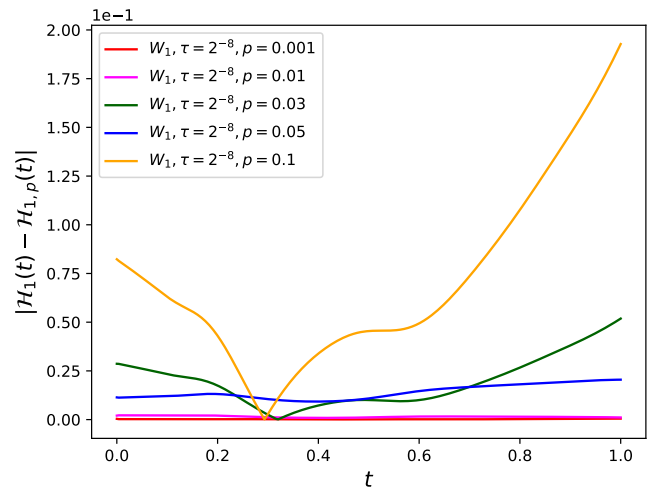

(b)

Figure 4: Experiment 1: (a) The exact source $\mathcal{H}_{1}$ and its numerical approximations, and (b) its corresponding absolute error, obtained for various levels of noise and using the mollifier $W_{1}$.

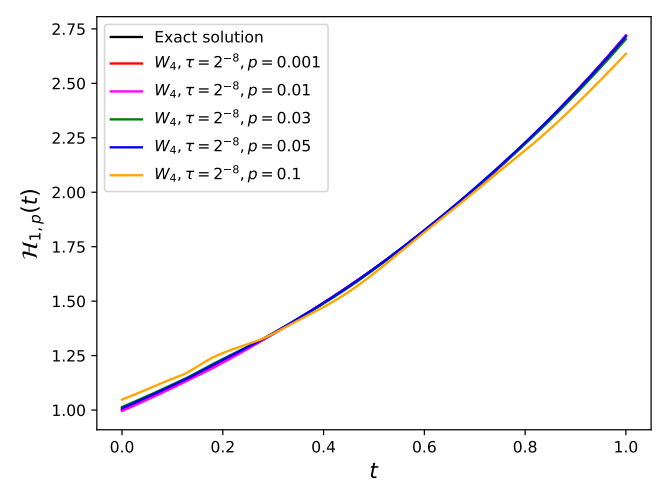

(a)

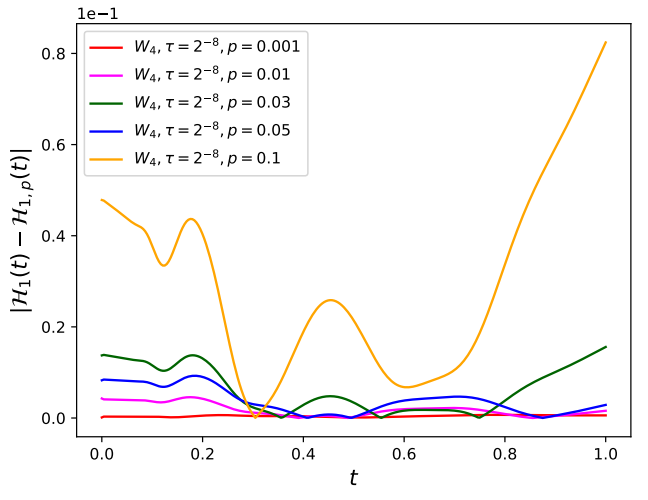

(b)

Figure 5: Experiment 1: (a) The exact source $\mathcal{H}_{1}$ and its numerical approximations, and (b) its corresponding absolute error, obtained for various levels of noise and using the mollifier $W_{4}$.

The results corresponding to Experiment 2 are depicted in Figures 6.7 and are similar to those obtained in the case of Experiment 1. Again, good numerical approximations have been obtained even for a large amount of noise ( $p=0.1$ and 0.2 ), with the mention that the shape of the source has been well-preserved. In [7], the authors reconstructed the source $\mathcal{H}_{2}$ from Dirichlet measured boundary data. If we compare the present results and those retrieved by Hasanov [7] for the same noise percentages, then the numerical approximations obtained herein are more accurate. 


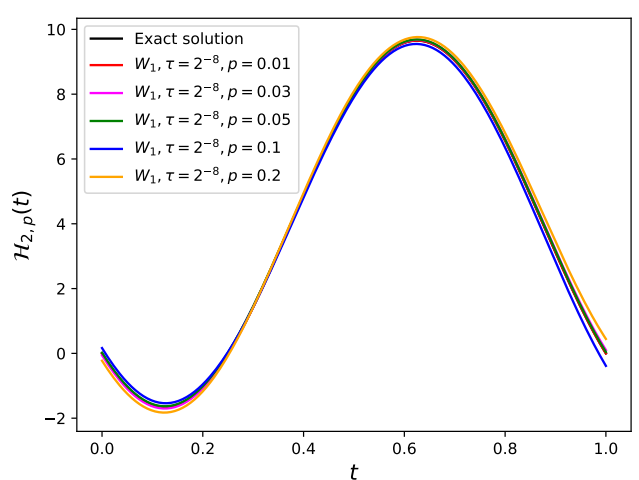

(a)

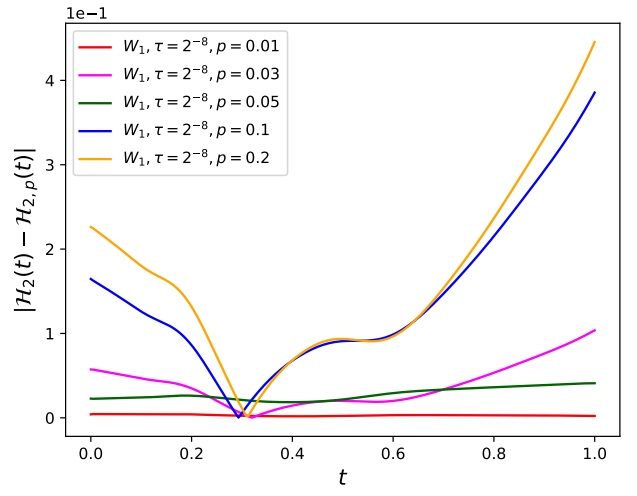

(b)

Figure 6: Experiment 2: (a) The exact source $\mathcal{H}_{2}$ and its numerical approximations, and (b) its corresponding absolute error, obtained for various levels of noise and using the mollifier $W_{1}$.

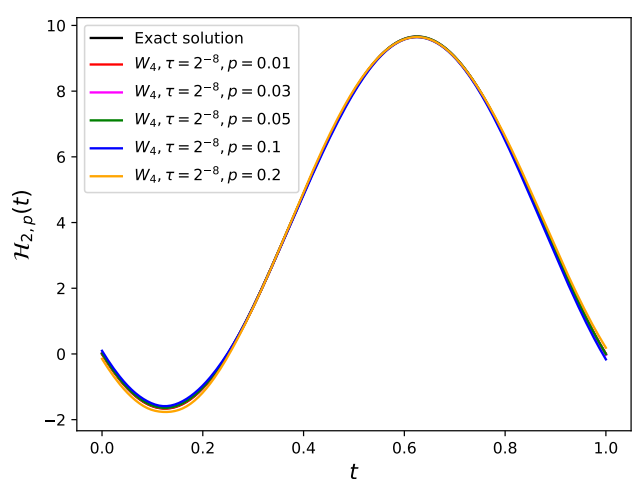

(a)

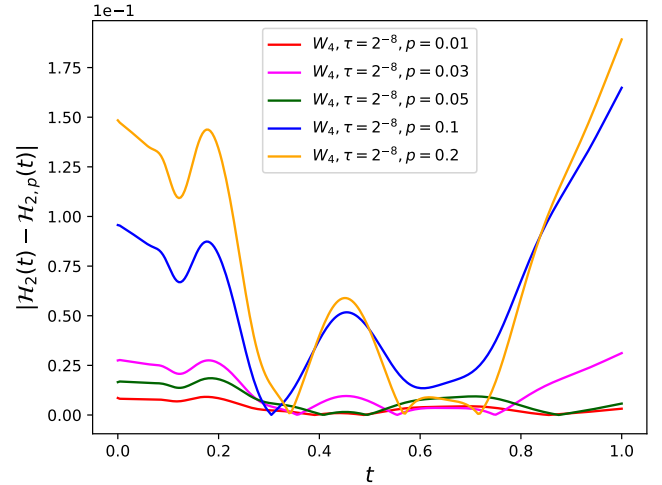

(b)

Figure 7: Experiment 2: (a) The exact source $\mathcal{H}_{2}$ and its numerical approximations, and (b) its corresponding absolute error, obtained for various levels of noise and using the mollifier $W_{2}$.

Finally, we emphasize that the numerical experiments performed in this section confirm the convergence rates obtained in the error estimates. Therefore, we may conclude that the proposed numerical algorithm gives an accurate numerical approximation for the unknown source even in the case of a regularized noisy measurement.

\section{Conclusion}

In this paper, an inverse source problem associated with a simply supported non-homogeneous EulerBernoulli beam has been investigated. More specifically, the theoretical and numerical determination of a time-dependent source from the knowledge of a so-called localized integral measurement has been studied. 
A convergent and stable algorithm based on Rothe's method has been proposed for the recovery of the missing source. Moreover, the uniqueness of a solution has been proved. The numerical experiments carried out herein have been implemented using the FEM. They validate the convergence and stability of the proposed procedure. Future work will be concerned with the multidimensional case and the implementation of additional experiments.

\section{Acknowledgments.}

Karel Van Bockstal has been supported by a postdoctoral fellowship of the Research Foundation - Flanders (106016/12P2919N) and partially supported by a grant of the Ministry of Research and Innovation, CNCS-UEFISCDI, project number PN-III-P4-ID-PCE-2016-0083, within PNCDI III. Liviu Marin has been supported by a grant of the Ministry of Research and Innovation, CNCS-UEFISCDI, project number PN-III-P4-ID-PCE-2016-0083, within PNCDI III.

[1] S. Nicaise and O. Zair. Determination of point sources in vibrating beams by boundary measurements: Identifiability, stability, and reconstruction results. Electronic Journal of Differential Equations (EJDE) [electronic only], 2004:Paper No. 20, 17 p., electronic only-Paper No. 20, 17 p., electronic only, 2004.

[2] A. Hasanov. Identification of an unknown source term in a vibrating cantilevered beam from final overdetermination. Inverse Problems, 25(11):115015, 2009.

[3] C.-S. Liu. A lie-group adaptive differential quadrature method to identify an unknown force in an euler-bernoulli beam equation. Acta Mechanica, 223(10):2207-2223, 2012.

[4] A. Kawano. Uniqueness in the identification of asynchronous sources and damage in vibrating beams. Inverse Problems, 30(6):065008, 2014

[5] A. Hasanov and O. Baysal. Identification of an unknown spatial load distribution in a vibrating cantilevered beam from final overdetermination. J. Inverse Ill-Posed Probl., 23(1):85-102, 2015.

[6] A. Hasanov and A. Kawano. Identification of unknown spatial load distributions in a vibrating eulerbernoulli beam from limited measured data. Inverse Problems, 32(5):055004, 2016.

[7] A. Hasanov and O. Baysal. Identification of unknown temporal and spatial load distributions in a vibrating eulerbernoulli beam from dirichlet boundary measured data. Automatica, 71:106-117, 2016.

[8] K. Van Bockstal. Identification of an unknown spatial load distribution in a vibrating beam or plate from the final state. Journal of Inverse and Ill-posed Problems, 0, 2019.

[9] M. Grimmonprez and M. Slodička. Full discretization of a nonlinear parabolic problem containing volterra operators and an unknown dirichlet boundary condition. Numerical Methods for Partial Differential Equations, 31(5):1444-1460, 2015.

[10] M. Grimmonprez and M. Slodička. Reconstruction of an unknown source parameter in a semilinear parabolic problem. $J$. Comput. Appl. Math., 289:331-345, 2015.

[11] L. Šeliga and M. Slodička. An inverse source problem for a damped wave equation with memory. J. Inverse Ill-Posed Problems, 24(2):111-122, 2016.

[12] K. Van Bockstal and M. Slodička. Recovery of a time-dependent heat source in one-dimensional thermoelasticity of type-III. Inverse Problems in Science and Engineering, 25(5):749-770, 2017.

[13] K. Van Bockstal and L. Marin. Recovery of a space-dependent vector source in anisotropic thermoelastic systems. Computer Methods in Applied Mechanics and Engineering, 321:269-293, 2017.

[14] K. Van Bockstal, M. Slodička, and F. Gistelinck. Identification of a memory kernel in a nonlinear integrodifferential parabolic problem. Applied Numerical Mathematics , 120:305-323, 2017.

[15] J. Nečas. Direct Methods in the Theory of Elliptic Equations. Springer Monographs in Mathematics, 2011. 
[16] R. B. Guenther and J. W. Lee. Partial Differential Equations of Mathematical Physics and Integral Equations. Dover books on mathematics. Dover Publications, United States of America, 1988.

[17] J. Kačur. Method of Rothe in evolution equations, volume 80 of Teubner Texte zur Mathematik. Teubner, Leipzig, 1985.

[18] E. Zeidler. Nonlinear Functional Analysis and its Applications II/A: Linear Monotone Operators. Springer, 1990.

[19] A. Kufner, O. John, and S. Fučík. Function Spaces. Monographs and textbooks on mechanics of solids and fluids. Noordhoff International Publishing, Leyden, 1977.

[20] A. Logg and G. N. Wells. DOLFIN: Automated Finite Element Computing. ACM Trans. Math. Software, 37(2):28, 2010.

[21] A. Logg, G.N. Wells, and J. Hake. DOLFIN: a C++/Python Finite Element Library, chapter 10. Springer, 2012.

[22] A. Logg, K-A. Mardal, G. N. Wells, et al. Automated Solution of Differential Equations by the Finite Element Method. Springer, 2012.

[23] M. S. Alnæs, J. Blechta, J. Hake, A. Johansson, B. Kehlet, A. Logg, C. Richardson, J. Ring, M. E. Rognes, and G. N. Wells. The FEniCS project version 1.5. Archive of Numerical Software, 3(100), 2015. 